\title{
Limit lamination theorem for H-disks
}

\section{William H. Meeks III ${ }^{1}$ - Giuseppe Tinaglia ${ }^{2}$}

Received: 4 November 2015 / Accepted: 17 April 2021 / Published online: 4 May 2021 (C) The Author(s) 2021

\begin{abstract}
We describe the lamination limits of sequences of compact disks $M_{n}$ embedded in $\mathbb{R}^{3}$ with constant mean curvature $H_{n}$, when the boundaries of these disks tend to infinity. This theorem generalizes to the non-zero constant mean curvature case Theorem 0.1 by Colding and Minicozzi (Ann Math $160: 573-615,2004)$ for minimal disks. We apply this theorem to prove the existence of a chord arc result for compact disks embedded in $\mathbb{R}^{3}$ with constant mean curvature; this chord arc result generalizes Theorem 0.5 by Colding and Minicozzi (Ann Math 167:211-243, 2008) for minimal disks.
\end{abstract}

Mathematics Subject Classification Primary 53A10; Secondary 49Q05 . $53 \mathrm{C} 42$

This material is based upon work for the NSF under Award No. DMS-1309236. Any opinions, findings, and conclusions or recommendations expressed in this publication are those of the authors and do not necessarily reflect the views of the NSF.

Giuseppe Tinaglia was partially supported by EPSRC Grant No. EP/M024512/1.

Giuseppe Tinaglia

giuseppe.tinaglia@kcl.ac.uk

William H. Meeks III

profmeeks@gmail.com

1 Mathematics Department, University of Massachusetts, Amherst, MA 01003, USA

2 Department of Mathematics, King's College London, London WC2R 2LS, UK 


\section{Introduction}

In this paper we apply results in [22-24] to obtain (after passing to a subsequence) minimal lamination limits for any sequence of compact disks $M_{n}$ embedded in $\mathbb{R}^{3}$ with constant mean curvature $H_{n}$, when the boundaries of these disks tend to infinity; see Theorem 1.1 below. This theorem is inspired by, and generalizes to the non-zero constant mean curvature setting, Theorem 0.1 by Colding and Minicozzi [7] and it is related to work in [3, 14, 15,26]. As an application of Theorem 1.1, we obtain a chord arc result for compact disks embedded in $\mathbb{R}^{3}$ with constant mean curvature that does not depend on the value of the mean curvature; this chord arc result is stated below in Theorem 1.2. Theorem 1.2 is inspired by and generalizes the chord arc result by Colding and Minicozzi for embedded minimal disks appearing in Theorem 0.5 of [9].

For clarity of exposition, we will call an oriented surface $M$ immersed in $\mathbb{R}^{3}$ an $H$-surface if it is embedded, connected and it has non-negative constant mean curvature $H$. We will call an $H$-surface an $H$-disk if the $H$-surface is homeomorphic to a closed unit disk in the Euclidean plane. Here $\mathbb{B}(R)$ denotes the open ball in $\mathbb{R}^{3}$ centered at the origin $\mathbf{0}$ of radius $R$ and for a point $p$ on a surface $\Sigma \subset \mathbb{R}^{3},\left|A_{\Sigma}\right|(p)$ denotes the norm of the second fundamental form of $\Sigma$ at $p$.

Theorem 1.1 (Limit lamination theorem for $H$-disks) Fix $\varepsilon>0$ and let $\left\{M_{n}\right\}_{n}$ be a sequence of $H_{n}$-disks in $\mathbb{R}^{3}$ containing the origin and such that $\partial M_{n} \subset$ $\left[\mathbb{R}^{3} \backslash \mathbb{B}(n)\right]$ and $\left|A_{M_{n}}\right|(\mathbf{0}) \geq \varepsilon$. Then, after replacing by some subsequence, exactly one of the following two statements hold.

A. The surfaces $M_{n}$ converge smoothly with multiplicity one or two on compact subsets of $\mathbb{R}^{3}$ to a helicoid $M_{\infty}$ containing the origin. Furthermore, every component $\Delta$ of $M_{n} \cap \mathbb{B}(1)$ is an open disk whose closure $\bar{\Delta}$ in $M_{n}$ is a compact disk with piecewise smooth boundary, and where the intrinsic distance in $M_{n}$ between any two points in $\bar{\Delta}$ is less than 10.

B. There are points $p_{n} \in M_{n}$ such that

$$
\lim _{n \rightarrow \infty} p_{n}=\mathbf{0} \text { and } \lim _{n \rightarrow \infty}\left|A_{M_{n}}\right|\left(p_{n}\right)=\infty,
$$

and the following hold:

(a) The surfaces $M_{n}$ converge to a foliation of $\mathbb{R}^{3}$ by planes and the convergence is $C^{\alpha}$, for any $\alpha \in(0,1)$, away from the line containing the origin and orthogonal to the planes in the foliation.

(b) There exists compact subdomains $\mathcal{C}_{n}$ of $M_{n},\left[M_{n} \cap \overline{\mathbb{B}}(1)\right] \subset \mathcal{C}_{n} \subset \mathbb{B}(2)$ and $\partial \mathcal{C}_{n} \subset \mathbb{B}(2) \backslash \overline{\mathbb{B}}(1)$, each $\mathcal{C}_{n}$ consisting of one or two disjoint disks, where each disk component has intrinsic diameter less than 3 and 
intersects $\mathbb{B}(1 / n)$. Moreover, each connected component of $M_{n} \cap \mathbb{B}(1)$ is an open disk whose closure in $M_{n}$ is a compact disk with piecewise smooth boundary.

As an application of Theorem 1.1 and its proof, we prove in Theorem $4.3 \mathrm{a}$ sharp chord arc result for $H$-disks (also see Remark 4.4); the following chord arc result for $H$-disks is a restatement of Eq. (10) in Theorem 4.3.

Theorem 1.2 (Chord arc property for $H$-disks) There exists $a C>1$ so that the following holds. Suppose that $\Sigma$ is an $H$-disk, $\mathbf{0} \in \Sigma$ and $R>r_{0}>0$. If the open intrinsic ball $B_{\Sigma}(\mathbf{0}, C R)$ of radius $C R$ centered at $\mathbf{0}$ is contained in $\Sigma \backslash \partial \Sigma$ and $\sup _{B_{\Sigma}\left(\mathbf{0},\left(1-\frac{\sqrt{2}}{2}\right) r_{0}\right)}\left|A_{\Sigma}\right|>r_{0}^{-1}$, then

$$
\frac{1}{3} \operatorname{dist}_{\Sigma}(x, \mathbf{0}) \leq|x| / 2+r_{0}, \text { for } x \in B_{\Sigma}(\mathbf{0}, R) .
$$

The proofs of the results described in this paper depend in an essential manner on the existence of extrinsic curvature estimates for disks embedded in $\mathbb{R}^{3}$ of non-zero constant mean curvature that appear in [23], as well as on a key extrinsic one-sided curvature estimate obtained in [24] and a weak cord arc result derived in [22]; these results from [22-24] are described in Sect. 2.

\section{Preliminaries}

Throughout this paper, we use the following notation. Given $a, b, R>0$, $p \in \mathbb{R}^{3}$ and $\Sigma$ a surface in $\mathbb{R}^{3}$ :

- $\mathbb{B}(p, R)$ is the open ball of radius $R$ centered at $p$.

- $\mathbb{B}(R)=\mathbb{B}(\mathbf{0}, R)$, where $\mathbf{0}=(0,0,0)$.

- For $p \in \Sigma, B_{\Sigma}(p, R)$ denotes the open intrinsic ball in $\Sigma$ of radius $R$.

- $C(a, b)=\left\{\left(x_{1}, x_{2}, x_{3}\right)\left|x_{1}^{2}+x_{2}^{2} \leq a^{2},\right| x_{3} \mid \leq b\right\}$.

- $A\left(r_{1}, r_{2}\right)=\left\{\left(x_{1}, x_{2}, 0\right) \mid r_{2}^{2} \leq x_{1}^{2}+x_{2}^{2} \leq r_{1}^{2}\right\}$.

We first introduce the notion of multi-valued graph, see [6] for further discussion. Intuitively, an $N$-valued graph is a simply-connected embedded surface covering an annulus such that over a neighborhood of each point of the annulus, the surface consists of $N$ graphs. The stereotypical infinite multi-valued graph is half of the helicoid, i.e., half of an infinite double-spiral staircase (Fig. 1).

Definition 2.1 (Multi-valued graph) Let $\mathcal{P}$ denote the universal cover of the punctured $\left(x_{1}, x_{2}\right)$-plane, $\left\{\left(x_{1}, x_{2}, 0\right) \mid\left(x_{1}, x_{2}\right) \neq(0,0)\right\}$, with global coordinates $(\rho, \theta)$. 


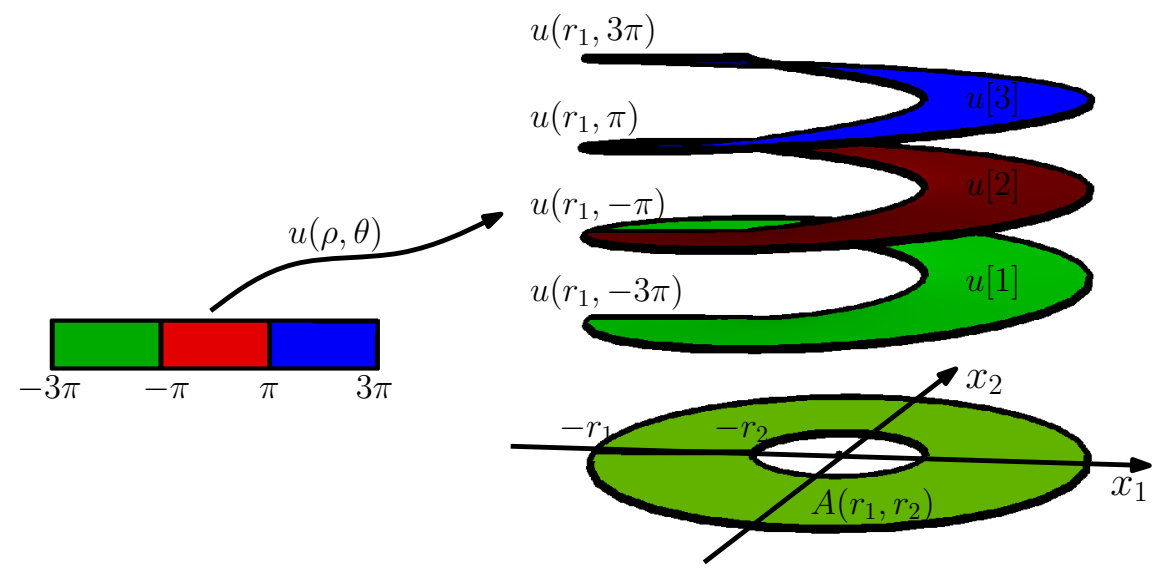

Fig. 1 A right-handed 3-valued graph

1. An $N$-valued graph over the annulus $A\left(r_{1}, r_{2}\right)$ is a single valued graph $u(\rho, \theta)$ over $\left\{(\rho, \theta)\left|r_{2} \leq \rho \leq r_{1},\right| \theta \mid \leq N \pi\right\} \subset \mathcal{P}$, if $N$ is odd, or over $\left\{(\rho, \theta) \mid r_{2} \leq \rho \leq r_{1},(-N+1) \pi \leq \theta \leq \pi(N+1)\right\} \subset \mathcal{P}$, if $N$ is even.

2. An $N$-valued graph $u(\rho, \theta)$ over the annulus $A\left(r_{1}, r_{2}\right)$ is called righthanded [lefthanded] if whenever it makes sense, $u(\rho, \theta)<u(\rho, \theta+2 \pi)$ $[u(\rho, \theta)>u(\rho, \theta+2 \pi)]$

3. The set $\left\{\left(r_{2}, \theta, u\left(r_{2}, \theta\right)\right), \theta \in[-N \pi, N \pi]\right\}$ when $N$ is odd (or $\left\{\left(r_{2}, \theta\right.\right.$, $\left.\left.u\left(r_{2}, \theta\right)\right), \theta \in[(-N+1) \pi,(N+1) \pi]\right\}$ when $N$ is even) is the inner boundary of the $N$-valued graph.

From Theorem 2.23 in [23] one obtains the following, detailed geometric description of an $\mathrm{H}$-disk with large norm of the second fundamental form at the origin. The precise meaning of certain statements below are made clear in [23] and we refer the reader to that paper for further details.

Theorem 2.2 Given $\varepsilon, \tau>0$ and $\bar{\varepsilon} \in(0, \varepsilon / 4)$ there exist constants $\Omega_{\tau}:=$ $\Omega(\tau), \omega_{\tau}:=\omega(\tau)$ and $G_{\tau}:=G(\varepsilon, \tau, \bar{\varepsilon})$ such that if $M$ is an $H$-disk, $H \in$ $\left(0, \frac{1}{2 \varepsilon}\right), \partial M \subset \partial \mathbb{B}(\varepsilon), \mathbf{0} \in M$ and $\left|A_{M}\right|(\mathbf{0})>\frac{1}{\eta} G_{\tau}$, for $\eta \in(0,1]$, then for any $p \in \overline{\mathbb{B}}(\mathbf{0}, \eta \bar{\varepsilon})$ that is a maximum of the function $\left|A_{M}\right|(\cdot)(\eta \bar{\varepsilon}-|\cdot|)$, after translating $M$ by $-p$, the following geometric description of $M$ holds:

On the scale of the norm of the second fundamental form $M$ looks like one or two helicoids nearby the origin and, after a rotation that turns these helicoids into vertical helicoids, $M$ contains a 3-valued graph u over $A(\varepsilon /$ $\left.\Omega_{\tau}, \frac{\omega_{\tau}}{\left|A_{M}\right|(\mathbf{0})}\right)$ with the norm of its gradient less than $\tau$ and with its inner boundary in $\mathbb{B}\left(10 \frac{\omega_{\tau}}{\left|A_{M}\right|(\mathbf{0})}\right)$.

Theorem 2.2 was inspired by the pioneering work of Colding and Minicozzi in the minimal case [5-8]; however in the constant positive mean curvature 
setting this description has led to a different conclusion, that is the existence of radius and curvature estimates stated below.

Theorem 2.3 (Extrinsic radius estimates, Theorem 3.4 in [23]) There exists an $\mathcal{R}_{0} \geq \pi$ such that for any $H$-disk $\mathcal{D}$,

$$
\sup _{p \in \mathcal{D}}\left\{d_{\mathbb{R}^{3}}(p, \partial \mathcal{D})\right\} \leq \frac{\mathcal{R}_{0}}{H} .
$$

Theorem 2.4 (Extrinsic curvature estimates, Theorem 3.5 in [23]) Given $\delta, \mathcal{H}>0$, there exists a constant $K_{0}(\delta, \mathcal{H})$ such that for any $H$-disk $\mathcal{D}$ with $H \geq \mathcal{H}$,

$$
\sup _{\left\{p \in \mathcal{D} \mid d_{\mathbb{R}^{3}}(p, \partial \mathcal{D}) \geq \delta\right\}}\left|A_{\mathcal{D}}\right| \leq K_{0}(\delta, \mathcal{H}) .
$$

Indeed since the plane and the helicoid are complete simply-connected minimal surfaces properly embedded in $\mathbb{R}^{3}$, a radius estimate does not hold in the minimal case. Moreover rescalings of a helicoid give a sequence of embedded minimal disks with arbitrarily large norm of the second fundamental form at points arbitrarily far from its boundary; therefore in the minimal setting, the curvature estimates also do not hold.

The next two results from [24] will be essential tools in the proof of Theorem 1.1.

Theorem 2.5 (Extrinsic one-sided curvature estimates for $H$-disks) There exist $\varepsilon \in\left(0, \frac{1}{2}\right)$ and $C \geq 2 \sqrt{2}$ such that for any $R>0$, the following holds. Let $\mathcal{D}$ be an $H$-disk such that

$$
\mathcal{D} \cap \mathbb{B}(R) \cap\left\{x_{3}=0\right\}=\varnothing \text { and } \partial \mathcal{D} \cap \mathbb{B}(R) \cap\left\{x_{3}>0\right\}=\varnothing .
$$

Then:

$$
\sup _{x \in \mathcal{D} \cap \mathbb{B}(\varepsilon R) \cap\left\{x_{3}>0\right\}}\left|A_{\mathcal{D}}\right|(x) \leq \frac{C}{R} .
$$

In particular, if $\mathcal{D} \cap \mathbb{B}(\varepsilon R) \cap\left\{x_{3}>0\right\} \neq \varnothing$, then $H \leq \frac{C}{R}$.

The next corollary follows immediately from Theorem 2.5 by a simple rescaling argument. It roughly states that we can replace the $\left(x_{1}, x_{2}\right)$-plane by any surface that has a fixed uniform estimate on the norm of its second fundamental form.

Corollary 2.6 Given an $a \geq 0$, there exist $\varepsilon \in\left(0, \frac{1}{2}\right)$ and $C_{a}>0$ such that for any $R>0$, the following holds. Let $\Delta$ be a compact immersed surface 
in $\mathbb{B}(R)$ with $\partial \Delta \subset \partial \mathbb{B}(R), \mathbf{0} \in \Delta$ and satisfying $\left|A_{\Delta}\right| \leq a / R$. Let $\mathcal{D}$ be an $H$-disk such that

$$
\mathcal{D} \cap \mathbb{B}(R) \cap \Delta=\varnothing \text { and } \partial \mathcal{D} \cap \mathbb{B}(R)=\varnothing .
$$

Then:

$$
\sup _{x \in \mathcal{D} \cap \mathbb{B}(\varepsilon R)}\left|A_{\mathcal{D}}\right|(x) \leq \frac{C_{a}}{R} .
$$

In particular, if $\mathcal{D} \cap \mathbb{B}(\varepsilon R) \neq \varnothing$, then $H \leq \frac{C_{a}}{R}$.

Next, we recall the notion of flux of an $H$-surface; see for instance [11,12, 25] for further discussions of this invariant.

Definition 2.7 Let $\gamma$ be a 1-cycle in an $H$-surface $M$. The $f u x$ of $\gamma$ is $\int_{\gamma}(H \gamma+$ $\xi) \times \dot{\gamma}$, where $\xi$ is the unit normal to $M$ along $\gamma$.

The flux of a 1-cycle in an $H$-surface $M$ is a homological invariant and we say that $M$ has zero flux if the flux of any 1-cycle in $M$ is zero; in particular, since the first homology group of a disk is zero, an $H$-disk has zero flux. Finally, we also recall the following definition.

Definition 2.8 Let $U$ be an open set in $\mathbb{R}^{3}$. We say that a sequence of surfaces $\{\Sigma(n)\}_{n \in \mathbb{N}} \subset U$, has locally bounded norm of the second fundamental form in $U$ if for every compact subset $B$ in $U$, the norms of the second fundamental forms of the surfaces $\Sigma(n) \cap B$ are uniformly bounded.

\section{Proof of the limit lamination theorem for $\boldsymbol{H}$-disks}

Proof of Theorem 1.1 Let $\left\{M_{n}\right\}_{n \in \mathbb{N}}$ be a sequence of constant mean curvature disks that satisfy the hypotheses of Theorem 1.1. The extrinsic radius estimates given in Theorem 2.3 imply $\lim _{n \rightarrow \infty} H_{n}=0$. We will break the proof into the following two cases.

Case A: $\left\{M_{n}\right\}_{n \in \mathbb{N}}$ has locally bounded norm of the second fundamental form in $\mathbb{R}^{3}$.

Case B: There is a compact set $B$ and the sequence $\left\{M_{n}\right\}_{n \in \mathbb{N}}$ has unbounded norm of the second fundamental form in $B$.

Note that if Case A holds, we will prove item A of Theorem 1.1 holds and if Case B holds, we will prove that item B of Theorem 1.1 holds.

Some of the arguments in the proofs of Cases A and B listed above are borrowed from arguments appearing in the proofs of similar Cases A and $\mathrm{B}$ in the proof of Proposition 3.1 in [24], where all the details are given. 
Consequently, the reader may wish to consult the proof of Proposition 3.1 in [24] before continuing to read what follows.

We will first prove that item A the theorem under the hypothesis that Case A holds.

Claim 3.1 A subsequence of $\left\{M_{n}\right\}_{n \in \mathbb{N}}$ converges with multiplicity one or two to a helicoid $\mathcal{H}=M_{\infty}$ containing $\mathbf{0}$.

Proof The proof of this claim follows the arguments in the beginning of the proof of Case A of Proposition 3.1 in [24]. Note that in Proposition 3.1 in [24] $M_{n}$ is denoted by $\Sigma(n)$. For the sake of completeness we outline the main arguments in the proof of this fact. First a standard compactness argument using the fact that the surfaces $M_{n}$ have uniformly bounded norm of the second fundamental form in balls of $\mathbb{R}^{3}$ implies that after replacing by a subsequence, the surfaces converge $C^{1, \alpha}$, for any $\alpha \in(0,1)$, to a minimal lamination $\mathcal{L}$ of $\mathbb{R}^{3}$, and since $\left|A_{M_{n}}\right|(\mathbf{0}) \geq \delta$, then the leaf $M_{\infty}$ of $\mathcal{L}$ passing through the origin $\mathbf{0}$ has the norm of its second fundamental form bounded from below by $\delta$ at $\mathbf{0}$. By Theorem 1.6 in [20], the limit leaves of the minimal lamination $\mathcal{L}$ form a closed set $\mathbf{C}$ of parallel planes and every non-flat leaf of $\mathcal{L}$, including $M_{\infty}$, is properly embedded in a component of the collection of open slabs and half-spaces that form the components of $\mathbb{R}^{3} \backslash \mathbf{C}$. For any non-planar leaf $L$ of $\mathcal{L}$ certain subdomains of the surfaces $M_{n}$ converge to it with multiplicity one or two, as $n$ tends to infinity; otherwise a higher order convergence would imply that $L$ is stable and hence planar, which is a contradiction. From the multiplicity one or two convergence of the $\left\{M_{n}\right\}_{n \in \mathbb{N}}$ to the non-flat leaf $L$, a curve lifting argument implies that $L$ has genus zero. By the properness of finite genus leaves of a minimal lamination of $\mathbb{R}^{3}$ (Theorem 7 in [16]), $L$ must be properly embedded in $\mathbb{R}^{3}$. Hence, all of the leaves of $\mathcal{L}$ are properly embedded. Since the leaf $M_{\infty}$ is not flat, then the strong halfspace theorem in [10] implies that $M_{\infty}$ is the only leaf in $\mathcal{L}$. As the surfaces $M_{n}$ converge (on compact subsets of $\mathbb{R}^{3}$ ) with multiplicity one or two to $M_{\infty}$, a standard lifting argument of curves on $M_{\infty}$ to the surfaces $M_{n}$ for $n$ large implies that $M_{\infty}$ has zero flux, since the $H_{n}$-disks $M_{n}$ have zero flux. Since properly embedded minimal surfaces in $\mathbb{R}^{3}$ with zero flux have one end [4], $M_{\infty}$ must have one end; hence $M_{\infty}$ is simply-connected. By the uniqueness of the helicoid [21], $M_{\infty}$ must be a helicoid (see also [2] for a proof). Therefore, we conclude that a subsequence of the original sequence $\left\{M_{n}\right\}_{n \in \mathbb{N}}$ converges with multiplicity one or two to a helicoid $\mathcal{H}=M_{\infty}$ containing $\mathbf{0}$.

Note that, without loss of generality, we may assume that the surfaces $M_{n}$ are transverse to the sphere $\partial \mathbb{B}(1)$. The reason for this is given by the following argument. By Sard's theorem, we may replace the original sequence by the homothetic surfaces $M_{n}^{\prime}=\left(1+\varepsilon_{n}\right) M_{n}$ with $\lim _{n \rightarrow \infty} \varepsilon_{n}=0$ such that each $M_{n}^{\prime}$ is transverse to $\partial \mathbb{B}(1)$. Then the proof that a subsequence of the surfaces 
$M_{n}^{\prime}$ has the desired convergence implies that the original related subsequence has the same type of convergence. Henceforth, we will assume that the surfaces $M_{n}$ are transverse to the sphere $\partial \mathbb{B}(1)$.

We now check that for $n$ large every component of $M_{n} \cap \overline{\mathbb{B}}(1)$ is a compact disk $M_{n}$ with boundary in $\partial \mathbb{B}(1)$. Otherwise, after replacing by a subsequence, for $n$ sufficiently large there exists a component $\bar{\Delta}_{n}$ of $M_{n} \cap \overline{\mathbb{B}}(1)$ that is a planar domain with more than one boundary component and let $\Gamma_{n}$ be one of its boundary components. Let $D_{n}$ denote the compact subdisk of $M_{n}$ with boundary $\Gamma(n) \subset M_{n} \cap \partial \mathbb{B}(1)$ and notice that $D_{n} \not \subset \bar{B}(1)$. Hence, there is a point $p_{n} \in D_{n}$ that has maximal distance $R_{n}>1$ from the origin. Since the boundary of $D_{n}$ lies in $\partial B(1)$ and $D_{n}$ lies in $\mathbb{R}^{3} \backslash \overline{\mathbb{B}}(1)$ near $\partial D_{n}$, then $p_{n}$ is an interior point of $D_{n}$ not contained in $\mathbb{B}(1)$ and $D_{n}$ lies inside the closed ball $\overline{\mathbb{B}}\left(R_{n}\right)$ and intersects $\partial \mathbb{B}\left(R_{n}\right)$ at the point $p_{n}$. By the mean curvature comparison principle applied at the point $p_{n}$, the constant mean curvature of $M_{n}$ is at least $1 / R_{n}$ and so, since the constant mean curvature values of the surfaces $M_{n}$ are tending to zero as $n$ goes to infinity, the interior points $p_{n} \in D_{n}$ are diverging to infinity in $\mathbb{R}^{3}$ as $n$ goes to infinity. Let $p$ be a point on the axis of $\mathcal{H}$ that is closest to the origin. Let $r_{0}=|p|$ be the distance from $p$ to the origin and let $E$ be the smooth compact disk $\mathcal{H} \cap \overline{\mathbb{B}}\left(p, r_{0}+2\right)$. Then because of the multiplicity one or two convergence of the surfaces $M_{n}$ to $\mathcal{H}$, for $n$ large, $M_{n}$ contains a unique smooth disk subdomain $M_{E}(n)$ that contains the origin and that is a normal graph of small $C^{0}$-norm over $E$, and in the case the multiplicity of convergence is two, $M_{n}$ contains another smooth disk subdomain $M_{E}^{\prime}(n)$ that does not contain the origin and that is a normal graph of small $C^{0}$-norm over $E$; furthermore, for $n$ sufficiently large, every point of $M_{n} \cap \mathbb{B}(1)$ is contained in $M_{E}(n) \cup M_{E}^{\prime}(n)$. In particular, for $n$ large, $\Delta_{n} \subset\left(M_{E}(n) \cup M_{E}^{\prime}(n)\right) \subset \mathbb{B}\left(p, r_{0}+3\right)$, and so $\Gamma(n) \subset M_{E}(n)$ or $\Gamma(n) \subset$ $M_{E}^{\prime}(n)$. If $\Gamma(n) \subset M_{E}(n)$ then it bounds in $M_{E}(n)$ a disk $D_{n}^{\prime}$, which must be the disk $D_{n}$; if $\Gamma(n) \subset M_{E}^{\prime}(n)$ then it bounds in $M_{E}^{\prime}(n)$ a disk $D_{n}^{\prime}$, which again must be the disk $D_{n}$. This is a contradiction since $D_{n} \not \subset \mathbb{B}\left(p, r_{0}+3\right)$ for $n$ large, but $D_{n}^{\prime} \subset\left(M_{E}(n) \cup M_{E}^{\prime}(n)\right) \subset \mathbb{B}\left(p, r_{0}+3\right)$. This proves that every component $\Delta_{n}$ of $M_{n} \cap \mathbb{B}(1)$ is an open subdisk of the disk $M_{n}$ and $\bar{\Delta}_{n}$ is a compact disk with boundary in $\partial \mathbb{B}(1)$.

To complete the proof of Theorem 1.1 when Case A holds, it remains to prove the upper bound of 10 on the intrinsic distances on $M_{n}$ between any two points on a fixed disk $\Delta_{n}$ component of $M_{n} \cap \mathbb{B}(1)$ (and then by the continuity of the intrinsic distance function on $M_{n}$, the same property holds for points in $\bar{\Delta}_{n}$ ). This property follows easily because it holds for the helicoid $\mathcal{H}$; this completes the proof of the theorem when Case A holds.

Assume now that Case B holds, i.e., the sequence $\left\{M_{n}\right\}_{n \in \mathbb{N}}$ does not have bounded norm of the second fundamental form in some compact subset $B \subset$ $\mathbb{R}^{3}$. We will prove that item (B) of Theorem 1.1 holds in this case. After 
replacing $\left\{M_{n}\right\}_{n \in \mathbb{N}}$ by a subsequence, there is a point $x \in \mathbb{R}^{3}$ closest to $\mathbf{0}$ such that there exist points $x_{n} \in M_{n}$ with $\left|A_{M_{n}}\right|\left(x_{n}\right)>n$ and $\lim _{n \rightarrow \infty} x_{n}=x$. After another replacement by a subsequence, a straightforward application of Theorem 2.2 produces a sequence of 3-valued graphs $G_{n} \subset M_{n}$ with inner boundaries near $x_{n}$ that converge to a plane $P_{x}$ passing through $x$ (see the proof of Claim 3.5 in [24] for additional details on the construction of the graphs $G_{n} \subset M_{n}$ that converge to $P_{x}$ ). Next choose a rotation $T: \mathbb{R}^{3} \rightarrow \mathbb{R}^{3}$ such that $T(x)=\mathbf{0}$ and such that the plane $T\left(P_{x}\right)$ is the $\left(x_{1}, x_{2}\right)$-plane, and replace the surfaces $M_{n}$ by the rotated surfaces $T\left(M_{n}\right)$. With an abuse of notation we will let $M_{n}$ denote the rotated surfaces $T\left(M_{n}\right)$ and note that these new surfaces may possibly not pass through the origin. Note that in Claim 3.3 we will prove that $x=\mathbf{0}$.

After this replacement of $M_{n}$ by $T\left(M_{n}\right), P_{0}$ is the $\left(x_{1}, x_{2}\right)$-plane. By having the three-valued graphs $G_{n}$ converging to $P_{0}$ play the role of $\Delta$ in Corollary 2.6, we obtain that the sequence of surfaces $\left\{M_{n}\right\}_{n \in \mathbb{N}}$ has locally bounded norm of the second fundamental form outside some closed solid vertical cone $\mathcal{C}_{\mathbf{0}}$ based at $\mathbf{0}$ of a certain fixed aperture determined only by Corollary 2.6. After replacing $\left\{M_{n}\right\}_{n \in \mathbb{N}}$ by a subsequence, we may assume that the sequence $\left\{M_{n}\right\}_{n \in \mathbb{N}}$ converges to a minimal lamination $\mathcal{L}$ of $\mathbb{R}^{3} \backslash \mathcal{S}$, where $\mathcal{S}$ is a smallest closed set such that the sequence $M_{n}$ has locally bounded norm of the second fundamental form in $\mathbb{R}^{3} \backslash \mathcal{S}$, and no subsequence of these surfaces has a smaller singular set of convergence; see for example, the proof of Claim 3.4 in [24] for the diagonal type argument leading to this statement, where $M_{n}$ is denoted by $\Sigma(n)$. By the previous discussion in this paragraph, through each point $y \in \mathcal{S}$ there passes a horizontal plane $P_{y}$ and $\mathcal{S} \cap\left[\mathbb{R}^{3} \backslash \mathcal{C}_{y}\right]=\varnothing$, where $\mathcal{C}_{y}$ is the vertical cone previously described but based at $y$ instead of at $\mathbf{0}$. As already observed, the one-sided curvature estimates given in Corollary 2.6 imply that $\mathcal{S} \cap P_{y}=\{y\}$.

Fix $p \in \mathcal{S}$. We claim that there exist points $p_{n} \in M_{n}$, with vertical tangent planes and such that $\lim _{n \rightarrow \infty} p_{n}=p$. Otherwise in some small neighborhood of $p, M_{n}$ admits a non-zero Jacobi function induced by the parallel Killing field $E_{3}=(0,0,1)$. This would give estimates for the norm of the second fundamental form of $M_{n}$ near $p$ which is a contradiction. We note that this geometric argument also implies that if $p_{n} \in M_{n}$ is a sequence of points with vertical tangent planes that is converging to a point $q \in \mathbb{R}^{3}$, then there exists a sequence of points $q_{n} \in M_{n}$ with $\lim _{n \rightarrow \infty} d_{M_{n}}\left(p_{n}, q_{n}\right)=0$ (that is arbitrarily close to $\left.p_{n}\right)$ and with $\lim _{n \rightarrow \infty}\left|A_{M_{n}}\right|\left(q_{n}\right)=\infty$. Otherwise, after replacing by a subsequence, the norm of the second fundamental form of $M_{n}$ would be bounded in a geodesic ball of fixed radius centered at $p_{n}$ and thus a small but fixed neighborhood $U_{n}$ of $p_{n}$ would be graphical over the vertical tangent plane $T_{p_{n}} M_{n}$. On the one hand, the sequence $U_{n}$ clearly can't converge to a horizontal plane. On the other hand, it cannot converge to $\mathcal{S}$ either since by 
playing the role of $\Delta$ in Corollary 2.6, it would imply a bound for the norm of the second fundamental form of $M_{n}$ at points nearby $U_{n}$. This contradiction implies that $q \in \mathcal{S}$. In other words, if $\mathcal{Z}$ denotes the set of points in $\mathbb{R}^{3}$ that are limits of points $p_{n} \in M_{n}$ with vertical tangent planes, then this discussion implies that $\mathcal{Z}=\mathcal{S}$.

Fix $p \in \mathcal{S}$. We claim that there exist points $p_{n} \in M_{n}$, with vertical tangent planes and such that $\lim _{n \rightarrow \infty} p_{n}=p$. Otherwise in some small neighborhood of $p, M_{n}$ admits a non-zero Jacobi function induced by the parallel Killing field $E_{3}=(0,0,1)$, which would give curvature estimates of $M_{n}$ near $p$ which is a contradiction. Also this geometric description gives that if $p_{n} \in M_{n}$, $p_{n}$ is a sequence of points with vertical tangent planes that is converging to a point $q \in \mathbb{R}^{3}$, then there exists a sequence of points $q_{n} \in M_{n}$ with $\lim _{n \rightarrow \infty} d_{M_{n}}\left(p_{n}, q_{n}\right)=0$ and with $\lim _{n \rightarrow \infty}\left|A_{M_{n}}\right|\left(q_{n}\right)=\infty$. Otherwise, after replacing by a subsequence, a geodesic ball of fixed radius centered at $p_{n}$ would satisfy curvature estimates and thus be a graph over a vertical plane. By Corollary 2.6, the existence of this graph would lead to a contradiction because such a graph cannot be contained in either $\mathcal{S}$ or in a horizontal plane. In particular, this implies that $q \in \mathcal{S}$. In other words, if $\mathcal{Z}$ denotes the set of points in $\mathbb{R}^{3}$ that are limits of points $p_{n} \in M_{n}$ with vertical tangent planes, then this discussion implies that $\mathcal{Z}=\mathcal{S}$.

The following claim is analogous to the statement of Corollary 3.8 in [24] and indeed the proof is the same by using the above observation that intrinsically close to a point where the tangent plane is vertical, there exist points where the norm of the second fundamental form is arbitrarily large. We refer the reader to the proof of Corollary 3.8 in [24] for details in the proof of the next result.

Claim 3.2 Given $\Lambda, R, \varepsilon, k>0$, there exists $N=N(\Lambda, R, \varepsilon, k) \in \mathbb{N}$ such that the following holds. Let $\mathcal{H}$ denote a vertical helicoid containing the origin with maximal absolute Gaussian curvature $\frac{1}{2}$ at the origin and let $p_{n} \in M_{n} \cap$ $\mathbb{B}(R)$ be a sequence of points where the tangent planes are vertical. For any $n>N,\left|A_{M_{n}}\right|\left(p_{n}\right)>k$ and there exists a rotation $T_{n}$ about the $x_{3}$-axis such that the intersection set $\left(\left|A_{M_{n}}\right|\left(p_{n}\right)\left[M_{n}-p_{n}\right]\right) \cap \mathbb{B}(\Lambda)$ consists of one or two connected components and each component consists of a normal graph $u$ over its projection to $T_{n}(\mathcal{H})$ or $-T_{n}(\mathcal{H})$ and $\|u\|_{C^{2}} \leq \varepsilon$.

Since $\mathcal{S}$ is a closed set in $\mathcal{C}_{0}$ and the mapping $\left.x_{3}\right|_{\mathcal{C}_{0}} \rightarrow \mathbb{R}$ is proper, it follows that $x_{3}(\mathcal{S})$ is a closed subset of $\mathbb{R}$ and so $\mathbb{R} \backslash x_{3}(\mathcal{S})$ is a possibly empty collection of pairwise disjoint open intervals together with perhaps one or two half-lines.

Claim 3.3 The intersection $\left\{x_{3}=t\right\} \cap \mathcal{S}$ is a single point for every $t \in \mathbb{R}$. In fact, the set $\mathcal{S}$ is a vertical line passing through the origin and $x=\mathbf{0}$. 
Proof We first prove that the intersection $\left\{x_{3}=t\right\} \cap \mathcal{S}$ is a single point for every $t \in \mathbb{R}$. Since $\left|\left\{x_{3}=t\right\} \cap \mathcal{S}\right| \leq 1$, it suffices to show that $\left\{x_{3}=t\right\} \cap \mathcal{S} \neq \varnothing$. Arguing by contradiction, suppose that for some $t_{0} \in \mathbb{R},\left\{x_{3}=t_{0}\right\} \cap \mathcal{S}=\emptyset$. The point $t_{0}$ is contained in a component, $I=(a, b) \subset\left[\mathbb{R} \backslash x_{3}(\mathcal{S})\right]$, where perhaps $a$ or $b$ is equal to $\pm \infty$ but not both. Without loss of generality, we may assume that $a \neq-\infty$ and $b \in(a, \infty]$ and define $W=x_{3}^{-1}((a, b))$; the set $W$ is either an open slab or an open half-space. Let $\Delta$ be the set of points in $\partial W \cap \mathcal{S}$. By the previous discussion, $\Delta$ contains exactly one point of $\mathcal{S}$ in each component of $\partial W$. Since the sequence $\left\{M_{n} \cap W\right\}_{n}$ has uniformly locally bounded norm of the second fundamental form in $W$ and $\lim _{n \rightarrow \infty} H_{n}=0$, the sequence converges to the minimal lamination $\mathcal{L}_{W}=\mathcal{L} \cap W$ of $W$. Again the one-sided curvature estimates in Theorem 2.5 and Corollary 2.6 give that $\left[\mathcal{L}_{W} \cup \partial W\right] \backslash \Delta$ is a minimal lamination of $\mathbb{R}^{3} \backslash \Delta$. Note that $\mathcal{L}_{W} \neq \varnothing$, otherwise after placing a horizontal plane in $W$ disjoint from $\mathcal{L}$ and arbitrarily close to $\Delta$, one could apply the one-sided curvature estimate in Theorem 2.5 to show that $\Delta=\varnothing$. As already observed, the closure $\overline{\mathcal{L}}_{W}$ of $\mathcal{L}_{W}$ in $\mathbb{R}^{3} \backslash \Delta$ has the structure of a minimal lamination of $\mathbb{R}^{3} \backslash \Delta$, and it is obtained by adding the leaves $\partial W \backslash \Delta$ to the lamination $\mathcal{L}_{W}$.

Following the arguments at the end of the proof of Proposition 3.1 in [24], it is straightforward to check that the leaves of the lamination $\overline{\mathcal{L}}_{W}$ have genus zero. Briefly, to see this property holds one first shows that none of the leaves in this lamination contained in $W$ are stable, which by the Stable Limit Leaf Theorem in $[17,18]$ implies every leaf $L$ of $\mathcal{L}_{W}$ is nonflat and proper in $W$ and the convergence of the surfaces $M_{n}$ to $L$ has multiplicity one or two. Finally, a curve lifting argument shows that $L$ has genus zero.

Let $L$ be a proper nonflat genus-zero leaf in $\mathcal{L}_{W}$. Since we can view $\overline{\mathcal{L}}_{W}$ to be a minimal lamination of $\mathbb{R}^{3}$ with a countable number of singularities (the singularities being in the finite set $\Delta$ ), then item 6 in Theorem 1.8 of [19] implies that the closure $\bar{L}$ of $L$ in $\mathbb{R}^{3}$ is a properly embedded minimal surface in $\mathbb{R}^{3}$ which lies on one side of one of the boundary planes of $W$. (In fact, to apply item 6 in Theorem 1.8 of [19] it suffices that the genus of $L$ is finite.) But $L$ is contained in a half-space, which contradicts the half-space theorem in [10]. This contradiction implies that for any $t \in \mathbb{R},\left\{x_{3}=t\right\} \cap \mathcal{S} \neq \varnothing$, and, as previously observed, then $\left\{x_{3}=t\right\} \cap \mathcal{S}$ is a single point.

Recall again that by the one-sided curvature estimates given in Theorem 2.5, for any $y \in \mathcal{S}, \mathcal{S} \cap\left[\mathbb{R}^{3} \backslash \mathcal{C}_{y}\right]=\varnothing$ where $\mathcal{C}_{y}$ is the vertical cone based at $y$ previously described. Therefore $\mathcal{S}$ is a Lipschitz curve parameterized by its $x_{3}$-coordinate and the sequence $\left\{M_{n}\right\}_{n \in \mathbb{N}}$ converges to a minimal foliation $\mathcal{L}$ of $\mathbb{R}^{3} \backslash \mathcal{S}$ by punctured horizontal planes. Since before the replacement of $M_{n}$ by the rotated surfaces $T\left(M_{n}\right)$ the norms of the second fundamental forms of the surfaces were bounded away from zero at $\mathbf{0}$, the nature of the $C^{\alpha}$ convergence of the surfaces $T\left(M_{n}\right)$ to the flat planes in $\mathcal{L}$ outside of $\mathcal{S}$ implies 
that $T(\mathbf{0}) \in \mathcal{S}$; hence, the originally chosen singular point $x$ of convergence "closest" to $\mathbf{0}$ must be $\mathbf{0}$ itself and therefore $T(\mathbf{0})=\mathbf{0}$.

We next check that the curve $\mathcal{S}$ is a vertical line passing through the origin. Let

$$
\Gamma_{n}^{R}=N_{n}^{-1}\left(\left\{x_{1}^{2}+x_{2}^{2}=1\right\}\right) \cap \mathbb{B}(R)
$$

where $N_{n}$ denotes the Gauss map of $M_{n}$. By the discussion before Claim 3.2, the set $\Gamma_{n}^{R}$ is nonempty and converges to $\mathcal{S} \cap \mathbb{B}(R)$ as $n$ goes to infinity. Therefore, it suffices to show that $\Gamma_{n}^{R}$ converges $C^{1}$, possibly with multiplicity, to the line segment $\{(0,0, t) \mid t \in(-R, R)\}$. This follows by Claim 3.2 because for $n$ large, $\Gamma_{n}^{R}$ is an analytic curve with tangent lines converging uniformly to the $x_{3}$-axis in balls centered at the origin of any fixed radius.

The previous claims complete the proof of part (a) of item B in the theorem. It remains to prove the last statement in the theorem.

Claim 3.4 Let $\Gamma_{n}=N_{n}^{-1}\left(\left\{x_{1}^{2}+x_{2}^{2}=1\right\}\right) \cap \mathbb{B}(3)$. Then $\Gamma_{n}$ consists of one or two analytic curves for $n$ large.

Proof By Claim 3.3 and its proof, the set $\Gamma_{n}$ is a possibly disconnected analytic curve that converges $C^{1}$ to the line segment obtained by intersecting the $x_{3^{-}}$ axis with $\mathbb{B}(3)$. Hence, it suffices to prove that $\Gamma_{n} \cap\left\{x_{3}=0\right\}$ consists of one or two points for $n$ large. Suppose that, after replacing by a subsequence, $p_{1}(n), p_{2}(n), p_{3}(n) \in \Gamma_{n} \cap\left\{x_{3}=0\right\}$ are three distinct points converging to $\mathbf{0}$ and let $\Gamma_{n}(i)$ be the connected component of $\Gamma_{n}$ containing $p_{i}(n), i=$ $1,2,3$. Without loss of generality, after applying small horizontal translations and rotations around the $x_{3}$-axis, we may assume that $p_{1}(n)=\mathbf{0}, p_{3}(n)=$ $\left(t_{n}, 0,0\right)$ and $\left|p_{2}(n)\right| \leq t_{n}$ satisfying $\lim _{n \rightarrow \infty} t_{n}=0$.

Consider the subsequence of rescaled disks $\Sigma_{n}=\frac{1}{t_{n}} M_{n}$ that contain the points $\mathbf{0}$ and $(1,0,0)$ and let $q(n)=\frac{1}{t_{n}} p_{2}(n)$. Note that $\mathbf{0},(1,0,0), q(n) \in$ $\Sigma_{n} \cap\left\{x_{3}=0\right\}$, the tangent planes to $\Sigma_{n}$ at $\mathbf{0}, q_{n}$ and $(1,0,0)$ are vertical and $|q(n)| \leq 1$. Claim 3.2 implies that a subsequence of the homothetically scaled surfaces $\left|A_{M_{n}}\right|(\mathbf{0}) M_{n}$ converges to a vertical helicoid $\mathcal{H}$ containing the $x_{3}$-axis. We are going to use what we have proven so far to analyze three exhaustive cases that would occur after replacing by a subsequence.

1. $\lim _{n \rightarrow \infty}\left|A_{\Sigma_{n}}\right|(\mathbf{0})=0$;

2. $\lim _{n \rightarrow \infty}\left|A_{\Sigma_{n}}\right|(\mathbf{0})=C \in(0, \infty)$;

3. $\lim _{n \rightarrow \infty}\left|A_{\Sigma_{n}}\right|(\mathbf{0})=\infty$.

If $\lim _{n \rightarrow \infty}\left|A_{\Sigma_{n}}\right|(\mathbf{0})=\lim _{n \rightarrow \infty} t_{n}\left|A_{M_{n}}\right|(\mathbf{0})=0$, then $\left|A_{M_{n}}\right|(\mathbf{0}) M_{n}$ converges to $\mathcal{H}$ with multiplicity greater than two. This is because $\left|p_{2}(n)\right| \leq t_{n}$ implies there are at least three points in $\left|A_{M_{n}}\right|(\mathbf{0}) M_{n} \cap\left\{x_{3}=0\right\}$ converging to the origin and having vertical tangent planes. However, a multiplicity greater 
than two convergence implies stability. Since the helicoid is not stable, we have obtained a contradiction when case 1 holds.

Next, assume that $\lim _{n \rightarrow \infty}\left|A_{\Sigma_{n}}\right|(\mathbf{0})=\lim _{n \rightarrow \infty} t_{n}\left|A_{M_{n}}\right|(\mathbf{0})=C \in$ $(0, \infty)$. Again, by Claim 3.2 we have that a subsequence of $\left|A_{M_{n}}\right|(\mathbf{0}) M_{n}$ converges to a vertical helicoid $\mathcal{H}$ containing the origin and

$$
\Sigma_{n}=\frac{1}{t_{n}} M_{n}=\frac{1}{t_{n}\left|A_{M_{n}}\right|(\mathbf{0})}\left|A_{M_{n}}\right|(\mathbf{0}) M_{n}
$$

must converge to the vertical helicoid $\frac{1}{C} \mathcal{H}$. However, this leads to a contradiction because $(1,0,0)$ is a point in $\Sigma_{n}$ with vertical tangent plane. This proves the claim when case 2 holds.

Finally, assume that $\lim _{n \rightarrow \infty}\left|A_{\Sigma_{n}}\right|(\mathbf{0})=\lim _{n \rightarrow \infty} t_{n}\left|A_{M_{n}}\right|(\mathbf{0})=\infty$. By our previous discussion, there exists a foliation $\mathcal{F}$ of $\mathbb{R}^{3}$ by planes and a line $\mathcal{S}$ orthogonal to the planes in $\mathcal{F}$ such that $\mathbf{0} \in \mathcal{S}$ and after replacing by a subsequence, the new sequence of surfaces $\Sigma_{n}$ converges $C^{\alpha}$, for $\alpha \in(0,1)$, to the foliation $\mathcal{F} \backslash \mathcal{S}$ in $\mathbb{R}^{3} \backslash \mathcal{S}$. We claim that $\mathcal{F}$ must be a foliation by vertical planes. If $(1,0,0) \notin \mathcal{S}$, then the claim is true because the tangent plane to $\Sigma_{n}$ at $(1,0,0)$ is vertical. If $(1,0,0) \in \mathcal{S}$ then, since $\mathbf{0} \in \mathcal{S}$ and $\mathcal{S}$ is a straight line orthogonal to the planes in the foliation, $\mathcal{S}$ is the $x_{1}$-axis and $\mathcal{F}$ is a foliation by vertical planes.

Recall that as $n$ goes to infinity, the boundary curves of the disks $M_{n}$ are converging to infinity and the constant values of their mean curvatures are going to zero. For $n$ sufficiently large, let $\widetilde{M}_{n} \subset M_{n}$ be the $H_{n}$-subdisk containing the origin with $\partial \widetilde{M}_{n} \subset \partial \mathbb{B}(1)$. Without loss of generality we can assume $H_{n} \in\left(0, \frac{1}{2}\right)$. Let $G_{1}, \omega_{1}, \Omega_{1}$ be the values of the functions $G_{\tau}, \omega_{\tau}, \Omega_{\tau}$ given by Theorem 2.2, after fixing $\varepsilon=\tau=1, \bar{\varepsilon}=\frac{1}{2}$. Note that since $\lim _{n \rightarrow \infty} t_{n}\left|A_{\widetilde{M}_{n}}\right|(\mathbf{0})=\infty$, for $n$ sufficiently large, $\left|A_{\widetilde{M}_{n}}\right|(\mathbf{0}) \geq \frac{1}{t_{n}} G_{1}$. Thus, when $n$ is sufficiently large, Theorem 2.2 and the fact that $M_{n}$ converges to a foliation of $\mathbb{R}^{3}$ minus the $x_{3}$-axis by horizontal planes, imply that there exist points $p_{n} \in \widetilde{M}_{n} \cap \mathbb{B}\left(\frac{t_{n}}{2}\right)$ such that the following holds: $\widetilde{M}_{n}-p_{n}$, and thus $M_{n}-p_{n}$, contains a 3 -valued graph $u_{n}$ over $A\left(1 / \Omega_{1}, \frac{\omega_{1}}{\left|A_{M_{n}}\right|(\mathbf{0})}\right)$ with the norm of its gradient less than 1 and with its inner boundary in $\mathbb{B}\left(10 \frac{\omega_{1}}{\left|A_{M_{n}}\right|(\mathbf{0})}\right)$.

Note that since $p_{n} \in \mathbb{B}\left(\frac{t_{n}}{2}\right)$, a subsequence of the translated and scaled surfaces $\Sigma_{n}^{\prime}=\frac{1}{t_{n}}\left[M_{n}-p_{n}\right]$ must converge to the translated foliation $\mathcal{F}^{\prime}=$ $\mathcal{F}-\mathbf{v}$ away from the translated singular set $\mathcal{S}^{\prime}=\mathcal{S}-\mathbf{v}$, where $\mathbf{v}=\lim _{n \rightarrow \infty} \frac{p_{n}}{t_{n}}$, $|\mathbf{v}| \leq \frac{1}{2}$. In particular, the leaves of $\mathcal{F}^{\prime}$ are vertical planes and the singular set $\mathcal{S}^{\prime}$ is a horizontal line perpendicular to the leaves of $\mathcal{F}^{\prime}$. However, for $n$ large, $\Sigma_{n}^{\prime}$ contains a 3-valued graph $u_{n}^{\prime}=\frac{1}{t_{n}} u_{n}$ over $A\left(1 / t_{n} \Omega_{1}, \frac{\omega_{1}}{t_{n}\left|A_{M_{n}}\right|(\mathbf{0})}\right)$ with the norm of its gradient less than 1 and with its inner boundary in $\mathbb{B}\left(10 \frac{\omega_{1}}{t_{n}\left|A_{M_{n}}\right|(\boldsymbol{0})}\right)$. Since $\lim _{n \rightarrow \infty} t_{n}=0$ and $\lim _{n \rightarrow \infty} t_{n}\left|A_{\widetilde{M}_{n}}\right|(\mathbf{0})=\infty$, this contradicts the fact 
that the leaves of $\mathcal{F}^{\prime}$ are punctured vertical planes. This contradiction finishes the proof of the claim.

The next claim follows from arguments similar to those in the proof of the last claim and by Claim 3.2, after taking $\Lambda=4$ and $\varepsilon$ sufficiently small.

Claim 3.5 After replacing by a subsequence, assume that the number of components in $\Gamma_{n}$ does not change. For any fixed $\theta_{0} \in[0,1)$, there exists an $N\left(\theta_{0}\right) \in \mathbb{N}$ such that, after replacing by a subsequence, the following holds for $n \geq N\left(\theta_{0}\right)$. For any $\theta \in\left[-\theta_{0}, \theta_{0}\right]$, let

$$
\gamma_{n}^{\theta}:=M_{n} \cap N_{n}^{-1}\left(\left\{x_{1}^{2}+x_{2}^{2}+x_{3}^{2}=1\right\} \cap\left\{x_{3}=\theta\right\}\right) \cap \mathbb{B}(3),
$$

then $\gamma_{n}^{\theta}$ consists of one or two disjoint analytic curves, the number of these curves does not depend upon the choice of $\theta$ (and so this number is the same as the number of components in $\Gamma_{n}$ ), the curves $\gamma_{n}^{\theta}$ can be parameterized smoothly by their $x_{3}$-coordinates and as $n$ goes to infinity the curves $\gamma_{n}^{\theta}$ and the sets $\cup_{\tau \in\left[-\theta_{0}, \theta_{0}\right]} \gamma_{n}^{\tau}$ converge to the interval $\{(0,0, t) \mid t \in(-3,3)\}$.

We next explain how to construct the set $\mathcal{C}_{n}$ consisting of one or two disk components described in part (b) of item B of Theorem 1.1. This explanation will complete the proof of the theorem.

Fix a number $\beta \in(0,1 / 3)$. Let us first suppose that, after choosing a subsequence, for all $n \in \mathbb{N}, \Gamma_{n}$ is a single curve, which we denote by $\gamma(n)$. The curve $\gamma(n)$ can be parameterized by its $x_{3}$-coordinates that lie in some interval $\left(-3+\varepsilon_{n}^{1}, 3-\varepsilon_{n}^{2}\right)$, where, as $n$ goes to infinity, the sequences of numbers $\varepsilon_{n}^{1}, \varepsilon_{n}^{2} \in[0, \beta)$ converge to zero and $\gamma(n)$ is $\beta$-close to the $x_{3}$-axis. Then, by Claim 3.2 and by Claim 3.5 with $\Lambda$ chosen sufficiently large and $\varepsilon$ chosen sufficiently small, there exists an $N(\beta) \in \mathbb{N}$ such that for $n \geq N(\beta)$ the following holds:

For each $t \in(-1-\beta, 1+\beta)$, the vertical tangent plane $T_{\gamma(n)(t)} M_{n}$ intersects $\left(M_{n} \backslash \gamma(n)(t)\right) \cap \overline{\mathbb{B}}(1+2 \beta)$ transversely, in a set containing two components that are arcs such that the closure of their union is an analytic curve $\alpha_{t}^{n}$ with $\gamma(n)(t) \in \alpha_{t}^{n}$ and $\alpha_{t}^{n}$ is $\beta$-close to a line segment contained in $\tau_{t}^{n}=T_{\gamma(n)(t)} M_{n} \cap\left\{x_{3}=t\right\} \cap \overline{\mathbb{B}}(1+3 \beta)$ in the $C^{1}$-norm.

For $n \geq N(\beta)$, define the following piecewise smooth disks:

$$
\begin{aligned}
& \mathcal{C}_{n}^{\beta}= \bigcup_{t \in(-1-\beta, 1+\beta)} \alpha_{t}^{n}, \quad F_{n}^{\beta}=\bigcup_{t \in(-1-2 \beta, 1+2 \beta)} \tau_{t}^{n}, \\
& \widehat{F}_{n}^{\beta}=\bigcup_{t \in(-1-2 \beta, 1+2 \beta)}\left[\tau_{t}^{n}-\left(x_{1}(\gamma(n)(t)), x_{2}(\gamma(n)(t)), 0\right)\right]
\end{aligned}
$$

Let $I_{n}: F_{n}^{\beta} \rightarrow \widehat{F}_{n}^{\beta}$ be the associated diffeomorphism induced by translating the horizontal line segment $\tau_{t}^{n}$ to the horizontal line segment $\tau_{t}^{n}-$ 
$\left(x_{1}(\gamma(n))(t), x_{2}(\gamma(n)(t)), 0\right)$ at the same height. Note that $I_{n}(\gamma(n)(t))=$ $(0,0, t)$ for all $t \in(-1-2 \beta, 1+2 \beta)$. Claim 3.2 and the proof of Claim 3.3 imply that $N(\beta)$ can be chosen large enough so that the following condition also holds:

For all unit length tangent vectors $v$ to $\tau_{t}^{n} \subset F_{n}^{\beta}$ or to the $\operatorname{arc} \gamma(n) \cap F_{n}^{\beta}$,

$$
\frac{1}{\left|\left(I_{n}\right)_{*}(v)\right|} \in[1,1+\beta]
$$

where $\left(I_{n}\right)_{*}(v)$ denotes the tangent vector of the related image smooth arcs.

Claim 3.2 implies that $N(\beta)$ can also be chosen large enough so that the following condition also holds:

For $n \geq N(\beta)$, there is a unique injective map $\Pi_{n}: \mathcal{C}_{n}^{\beta} \rightarrow F_{n}^{\beta}$ defined at a point $(x, y, z) \in \alpha_{t}^{n}$ by

$$
\Pi_{n}((x, y, z))=(x, y, t) .
$$

The map $\Pi_{n}$ is smooth at points in $\mathcal{C}_{n}^{\beta} \backslash \gamma(n), \Pi_{n}$ restricted to $\gamma(n) \cap \mathcal{C}_{n}^{\beta}$ is the identity function and for all unit tangent vectors $v$ to the pair of arcs $\alpha_{t}^{n} \backslash\{\gamma(n)(t)\}$ or to $\gamma(n) \cap \mathcal{C}_{n}^{\beta}$,

$$
\frac{1}{\left|\left(\Pi_{n}\right)_{*}(v)\right|} \in[1,1+\beta]
$$

where $\left(\Pi_{n}\right)_{*}(v)$ denotes the tangent vector of the related image smooth arcs.

We next define a distance function $D$ on $\mathbb{R}^{3}$ that makes it into a metric space and that is useful for estimating distances $D$ between pairs of points in the disks $\mathcal{C}_{n}^{\beta}$, see Fig. 2.

Definition 3.6 Let $x=\left(x_{1}, x_{2}, x_{3}\right), y=\left(y_{1}, y_{2}, y_{3}\right)$ be points in $\mathbb{R}^{3}$.

1. If $x_{3}=y_{3}$, then

$$
D(x, y)=\sqrt{\left(y_{1}-x_{1}\right)^{2}+\left(y_{2}-x_{2}\right)^{2}} .
$$

2. Otherwise,

$$
D(x, y)=\sqrt{x_{1}^{2}+x_{2}^{2}}+\left|x_{3}-y_{3}\right|+\sqrt{y_{1}^{2}+y_{2}^{2}} .
$$

Given a point $p \in \mathbb{R}^{3}$, we let $B^{D}(p, R)$ denote the open ball center at $p$ of radius $R$. 


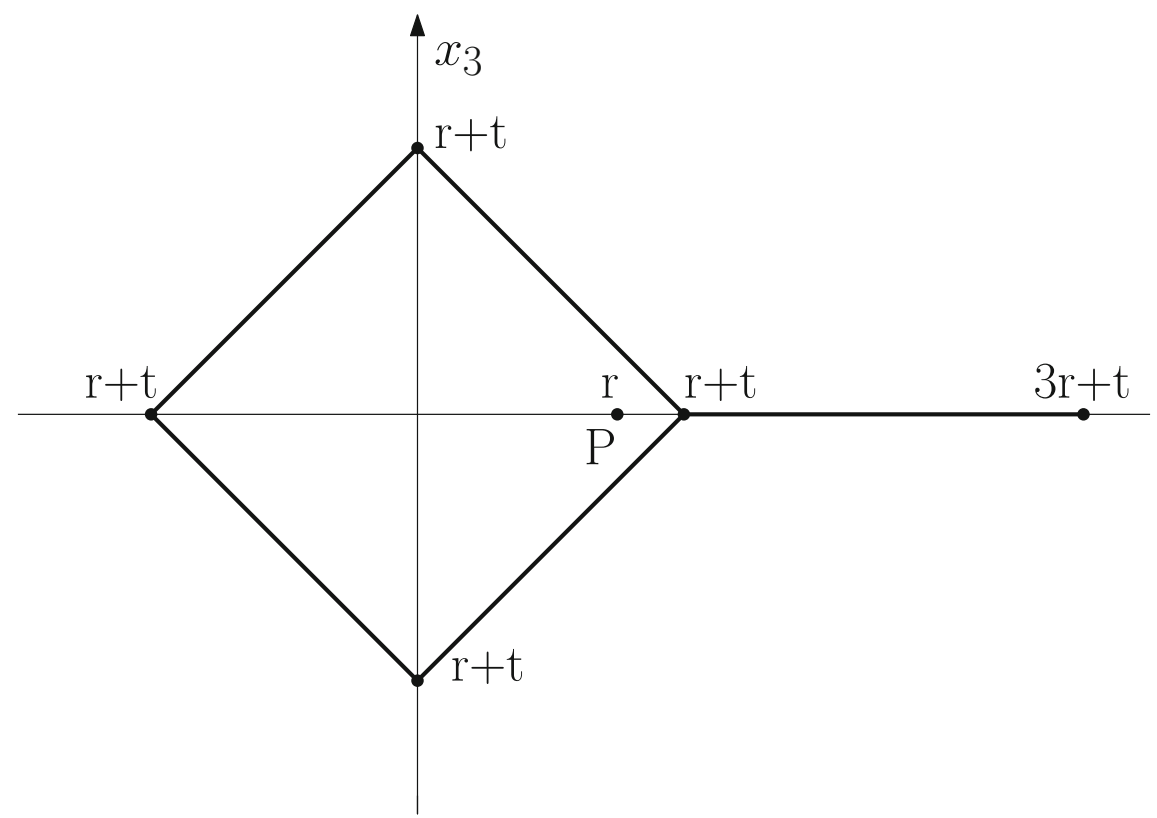

Fig. 2 The point $P$ is at distance $r$ from the $x_{3}$-axis. Given $t>0$, the thick line represents a vertical section of the topological boundary of $B^{D}(P, 2 r+t)$ containing $P$ and the $x_{3}$-axis. For $s \in(0, r+t]$, the intersection $B^{D}(P, 2 r+t) \cap\left\{x_{3}=x_{3}(P) \pm s\right\}$ consists of an open disk of radius $r+t-s$ centered at $\left(0,0, x_{3}(P) \pm s\right)$. The intersection $B^{D}(P, 2 r+t) \cap\left\{x_{3}=x_{3}(P)\right\}$ consists of the open disk centered at $P$ of radius $2 r+t$. It is easy to see that if $Q \notin B^{D}(P, 2 r+t)$ then $|Q-P| \geq \frac{\sqrt{2}}{2} t$

Geometrically speaking, this distance function is defined at a pair of points $p_{1}, p_{2} \in \mathbb{R}^{3}$ that lie on a horizontal line that intersects the $x_{3}$-axis to be equal to the length of the line segment between them. Otherwise it is equal to the length of the piecewise polygonal arc formed by the horizontal line segment joining $p_{1}$ to the $x_{3}$-axis, the horizontal line segment joining $p_{2}$ to the $x_{3}$ axis and the line segment in the $x_{3}$-axis that joins the end points of these two horizontal line segments. Note that $D$ induces a metric space structure with distance function $D_{\widehat{F}_{n}^{\beta}}$ on $\widehat{F}_{n}^{\beta}$ that is greater than or equal to the Riemannian distance function on the surface $\widehat{F}_{n}^{\beta}$.

Let $x, y \in \mathcal{C}_{n}^{\beta}$ be distinct points. If $x, y$ lie on the same $\alpha_{t}^{n}$ arc in $\mathcal{C}_{n}^{\beta}$, then let $c_{x, y}^{\beta, n}$ be the subarc with end points $x, y$. Otherwise, consider the embedded piecewise smooth path $c_{x, y}^{\beta, n}$ in $\mathcal{C}_{n}^{\beta}$ formed by the unique two subarcs in the $\alpha_{t}^{n}$-type curves joining, respectively, the points $x, y$ to points in $\gamma(n)$ together with the subarc in $\gamma(n)$ that joins the respective end points of these arcs in $\gamma(n)$; the reader should note the similarity of the construction of this piecewise smooth path joining $x$ and $y$ with the construction of the piecewise polygonal 
arc in the previous paragraph that joins points $p_{1}, p_{2}$ in $\mathbb{R}^{3}$ and where now $\gamma(n)$ plays the role of the $x_{3}$-axis. Let

$$
G_{n}^{\beta}: \mathcal{C}_{n}^{\beta} \rightarrow \widehat{F}_{n}^{\beta}, \text { where } G_{n}^{\beta}:=I_{n}^{\beta} \circ \Pi_{n}^{\beta},
$$

and given $x, y \in \mathcal{C}_{n}^{\beta}$, let $C_{x, y}^{\beta, n}$ denote the embedded piecewise smooth path $G_{n}^{\beta}\left(c_{x, y}^{\beta, n}\right)$ that has end points $G_{n}^{\beta}(x), G_{n}^{\beta}(y) \in \mathbb{B}(1+4 \beta)$. By Eqs. (4) and (5),

$$
\operatorname{Length}\left(c_{x, y}^{\beta, n}\right) \in\left[\operatorname{Length}\left(C_{x, y}^{\beta, n}\right),(1+\beta)^{2} \operatorname{Length}\left(C_{x, y}^{\beta, n}\right)\right] .
$$

Note that Length $\left(C_{x, y}^{\beta, n}\right)=D\left(G_{n}^{\beta}(x), G_{n}^{\beta}(y)\right)$. An elementary calculation shows that for any points $X, Y \in \overline{\mathbb{B}}(R), D(X, Y) \leq 2 \sqrt{2} R$. Hence, by Eq. (7), we have the estimate

$$
\text { Length }\left(c_{x, y}^{\beta, n}\right) \leq(1+\beta)^{2} \operatorname{Length}\left(C_{x, y}^{\beta, n}\right) \leq(1+\beta)^{2} 2(1+4 \beta) \sqrt{2} .
$$

Since as $n$ goes to infinity, $\beta$ can be chosen arbitrarily small, then for $n$ sufficiently large, we can assume that $(1+\beta)^{2} 2(1+4 \beta) \sqrt{2}<3$. With this choice of $\beta$,

$$
\text { Length }\left(c_{x, y}^{\beta, n}\right)<3
$$

and thus, the intrinsic diameter of $\mathcal{C}_{n}^{\beta}$ is less than 3 for $n$ sufficiently large. Henceforth, we will assume that $\beta$ is chosen sufficiently small and $n$ is chosen sufficiently large so that this inequality holds.

In the case being considered where $\Gamma_{n}$ is a single curve, after replacing by a sequence, define $\mathcal{C}_{n}:=\mathcal{C}_{n}^{\beta}$ and note that since $\mathbf{0} \in C_{n}, \mathcal{C}_{n} \cap \mathbb{B}(1 / n) \neq \varnothing$. By construction, $\partial \mathcal{C}_{n} \subset\left[\overline{\mathbb{B}}(1+2 \beta) \backslash \overline{\mathbb{B}}\left(1+\frac{2}{3} \beta\right)\right] \subset\left[\mathbb{R}^{3} \backslash \overline{\mathbb{B}}\left(1+\frac{2}{3} \beta\right)\right]$.

It remains to prove that for $n$ sufficiently large, every component $\Delta_{n}$ of $M_{n} \cap \mathbb{B}(1)$, and its closure $\bar{\Delta}_{n}$, is a disk that is contained in $\mathcal{C}_{n}$. We first prove that $\Delta_{n}$ is contained in $\mathcal{C}_{n}$. Once this is proved, the fact that $\Delta_{n}$ and its closure are disks with piecewise smooth boundary follows by using the same arguments as in the proof of item A of the theorem.

Arguing by contradiction, suppose that after choosing a subsequence, for all $n \in \mathbb{N}$, there is a component $\Delta_{n}$ of $M_{n} \cap \mathbb{B}(1)$ that is not contained in $\mathcal{C}_{n}$. Let $\widehat{\Delta}_{n}$ be the connected component of $M_{n} \cap \mathbb{B}\left(1+\frac{1}{2} \beta\right)$ containing $\Delta_{n}$. By elementary separation property, using that $\mathcal{C}_{n}$ is a disk disjoint from $\Delta_{n}$ and with $\partial \mathcal{C}_{n} \subset\left[\mathbb{R}^{3} \backslash \overline{\mathbb{B}}\left(1+\frac{2}{3} \beta\right)\right]$, it follows that $\widehat{\Delta}_{n}$ is also disjoint from $\mathcal{C}_{n}$. By the construction of $\mathcal{C}_{n}$, Claim 3.5 implies that given $\theta_{0} \in[0,1)$, if $n$ is sufficiently large then the set $\left(\bigcup_{\theta \in\left[-\theta_{0}, \theta_{0}\right]} \gamma_{n}^{\theta}\right) \cap \overline{\mathbb{B}}\left(1+\frac{1}{2} \beta\right)$ is contained in $\mathcal{C}_{n}$. Since $\widehat{\Delta}_{n}$ is disjoint from $\mathcal{C}_{n}$, we conclude that given $\varepsilon>0$ there exists $N(\varepsilon) \in \mathbb{N}$ such that if $n \geq N(\varepsilon)$, for any point $p \in \widehat{\Delta}_{n},|\langle N(p),(0,0,1)\rangle| \geq 1-\varepsilon$. Since 
$\widehat{\Delta}_{n}$ contains points in $\mathbb{B}(1)$ and has its boundary contained in $\partial \mathbb{B}\left(1+\frac{1}{2} \beta\right)$, if $\varepsilon$ is chosen sufficiently small depending on $\beta$, then $\widehat{\Delta}_{n}$ must intersect $\gamma(n)$ when $n$ is sufficiently large, contradicting embeddedness. This contradiction proves that the disk $\Delta_{n}$ must be contained in $\mathcal{C}_{n}$, and completes the proof of the theorem in the special case that $\Gamma_{n}$ has one component.

Suppose now that $\Gamma_{n}$ has exactly two components for all $n$. Let $\Gamma_{n} \cap \overline{\mathbb{B}}(1+$ $\beta)=\gamma_{1}(n) \cup \gamma_{2}(n)$, with $\mathbf{0} \in \gamma_{1}(n)$. Since the arcs $\gamma_{2}(n)$ converge to the intersection of the $x_{3}$-axis with the ball $\mathbb{B}(1+\beta)$, after replacing by a subsequence, $\gamma_{2}(n) \cap \mathbb{B}\left(\frac{1}{n}\right) \neq \varnothing$. As in the just considered case, we can construct a disk $\mathcal{C}_{n}^{\beta}(1)$ passing through the origin and that contains the "axis" $\gamma_{1}(n)$ and the intrinsic diameter of $\mathcal{C}_{n}^{\beta}(1)$ is less than 3 . After choosing a possibly smaller value of $\beta$, one can also construct a similarly defined "ruled" disk $\mathcal{C}_{n}^{\beta}(2) \subset \overline{\mathbb{B}}(1+2 \beta)$ with "axis" $\gamma_{2}(n)$ and the intrinsic diameter of $\mathcal{C}_{n}^{\beta}(2)$ is also less than 3 . Note that in this second case the disks $\mathcal{C}_{n}^{\beta}(1), \mathcal{C}_{n}^{\beta}(2)$ are also pairwise disjoint. The proof that every component of $M_{n} \cap \mathbb{B}(1)$ is a disk whose closure in $M_{n}$ is a piecewise smooth compact disk contained in $\mathcal{C}_{n}=\mathcal{C}_{n}^{\beta}(1) \cup \mathcal{C}_{n}^{\beta}(2)$ is the same as the proof when $\Gamma_{n}$ is a single curve. This last observation completes the proof of Theorem 1.1

\section{Applications of the main theorem}

\subsection{Chord-arc property}

In this section we prove the chord arc result stated in Theorem 1.2. Before doing so, we need to recall a result from [22]. In [22], we applied the onesided curvature estimate in Theorem 2.5 to prove a relation between intrinsic and extrinsic distances in an $H$-disk, which can be viewed as a weak chord arc property. This result was motivated by and generalizes a previous result, Proposition 1.1 in [9], by Colding-Minicozzi for 0 -disks. We begin by making the following definition.

Definition 4.1 Given a point $p$ on a surface $\Sigma \subset \mathbb{R}^{3}, \Sigma(p, R)$ denotes the closure of the component of $\Sigma \cap \mathbb{B}(p, R)$ passing through $p$.

Theorem 4.2 (Weak chord arc property, Theorem 1.2 in [22]) There exists a $\delta_{1} \in\left(0, \frac{1}{2}\right)$ such that the following holds.

Let $\Sigma$ be an $H$-disk in $\mathbb{R}^{3}$. Then for all intrinsic closed balls $\bar{B}_{\Sigma}(x, R)$ in $\Sigma \backslash \partial \Sigma$ :

1. $\Sigma\left(x, \delta_{1} R\right)$ is a disk with piecewise smooth boundary $\partial \Sigma\left(\mathbf{0}, \delta_{1} R\right) \subset$ $\partial \mathbb{B}\left(\delta_{1} R\right)$.

2. $\Sigma\left(x, \delta_{1} R\right) \subset B_{\Sigma}\left(x, \frac{R}{2}\right)$. 
Theorem 1.2 is Eq. (10) of this result.

Theorem 4.3 Given $\varepsilon>0$, there exists a $C_{\varepsilon}>1$ so that the following holds. Suppose that $\Sigma$ is an $H$-disk, $\mathbf{0} \in \Sigma$ and $R>r_{0}>0$. If $B_{\Sigma}\left(\mathbf{0}, C_{\varepsilon} R\right) \subset \Sigma \backslash \partial \Sigma$ and $\sup _{B_{\Sigma}\left(\mathbf{0},\left(1-\frac{\sqrt{2}}{2}\right) r_{0}\right)}\left|A_{\Sigma}\right| \geq r_{0}^{-1}$, then

$$
\operatorname{dist}_{\Sigma}(x, \mathbf{0})<(1+\varepsilon)\left(\sqrt{2}|x|+2 r_{0}\right), \text { for } x \in B_{\Sigma}(\mathbf{0}, R) .
$$

In particular, for $\varepsilon$ chosen sufficiently small, there exists a $C_{\varepsilon}$ so that

$$
\frac{1}{3} \operatorname{dist}_{\Sigma}(x, \mathbf{0})<|x| / 2+r_{0}, \text { for } x \in B_{\Sigma}(\mathbf{0}, R) .
$$

Remark 4.4 The constants in Eq. (9) are sharp in the following sense. For every $k \in \mathbb{N}$, there exists a helicoid $\Sigma$ with $\mathbf{0} \in \Sigma$ satisfying the following properties:

- $\sup _{B_{\Sigma}\left(\mathbf{0}, 1-\frac{\sqrt{2}}{2}\right)}\left|A_{\Sigma}\right|=1$,

- there exists points $x, y \in \Sigma$, such that

$$
\begin{aligned}
\operatorname{dist}_{\Sigma}(x, \mathbf{0}) & >\left(\sqrt{2}-\frac{1}{k}\right)|x|, \\
\operatorname{dist}_{\Sigma}(y, \mathbf{0}) & >2-\frac{1}{k} .
\end{aligned}
$$

Proof Arguing by contradiction, suppose there exists $\delta>0$ such that for any $n \in \mathbb{N}$ the following holds. There exist an $H_{n}$-disk $\Sigma_{n}$ with $\mathbf{0} \in \Sigma_{n}$, and $R_{n}>$ $r_{n}>0$, such that $B_{\Sigma_{n}}\left(\mathbf{0}, n^{2} R_{n}\right) \subset \Sigma_{n} \backslash \partial \Sigma_{n}, \sup _{B_{\Sigma_{n}}\left(\mathbf{0},\left(1-\frac{\sqrt{2}}{2}\right) r_{n}\right)}\left|A_{\Sigma_{n}}\right| \geq r_{n}^{-1}$, and

$$
\operatorname{dist}_{\Sigma_{n}}\left(x_{n}, \mathbf{0}\right) \geq(1+\delta)\left(\sqrt{2}\left|x_{n}\right|+2 r_{n}\right)
$$

for some $x_{n} \in B_{\Sigma_{n}}\left(\mathbf{0}, R_{n}\right)$.

Abusing the notation, let $\Sigma_{n}$ denote the rescaled surfaces $\frac{1}{n R_{n}} \Sigma_{n}$ and let $r_{n}$ denote $\frac{r_{n}}{n R_{n}}$. Then for the new sequence of disks $\Sigma_{n}$ the following holds. The intrinsic ball $B_{\Sigma_{n}}(\mathbf{0}, n)$ is contained in $\Sigma_{n} \backslash \partial \Sigma_{n}, \mathbf{0} \in \Sigma_{n}$,

$$
\sup _{B_{\Sigma_{n}}\left(\mathbf{0},\left(1-\frac{\sqrt{2}}{2}\right) r_{n}\right)}\left|A_{\Sigma_{n}}\right| \geq r_{n}^{-1},
$$

where $r_{n}<\frac{1}{n}$ and

$$
\operatorname{dist}_{\Sigma_{n}}\left(y_{n}, \mathbf{0}\right) \geq(1+\delta)\left(\sqrt{2}\left|y_{n}\right|+2 r_{n}\right)
$$


for some $y_{n} \in B_{\Sigma_{n}}(\mathbf{0}, 1 / n)$.

Theorem 4.2 implies that the surfaces $\widetilde{\Sigma}_{n}=\Sigma_{n}\left(\mathbf{0}, \delta_{1} n\right)$ are disks with piecewise smooth boundary $\partial \widetilde{\Sigma}_{n} \subset \partial \mathbb{B}\left(\delta_{1} n\right)$ and therefore, after choosing a subsequence, we can assume that $\partial \widetilde{\Sigma}_{n} \subset\left[\mathbb{R}^{3} \backslash \mathbb{B}(n)\right]$.

Since by Eq. (11), $\sup _{B_{\widetilde{\Sigma}_{n}}\left(\mathbf{0},\left(1-\frac{\sqrt{2}}{2}\right) r_{n}\right)}\left|A_{\widetilde{\Sigma}_{n}}\right| \geq r_{n}^{-1} \geq n$, we can apply the arguments in the proof of Case B of Theorem 1.1 to the sequence of constant mean curvature disks $\widetilde{\Sigma}_{n}$. Once again, abusing the notation, we let $\Sigma_{n}$ denote $\widetilde{\Sigma}_{n}$. Theorem 1.1 implies that, after choosing a subsequence, there exists a straight line $\mathcal{S}$ going through the origin, such that the sequence of surfaces $\Sigma_{n}$ converges to a minimal foliation of $\mathbb{R}^{3} \backslash \mathcal{S}$ by planes. Without loss of generality, we will assume these planes are horizontal and $\mathcal{S}$ is the $x_{3}$-axis.

By the proof of part B of Theorem 1.1, there is a set $\Gamma_{n} \subset \Sigma_{n} \cap \mathbb{B}(3)$ that consists of one or two analytic arcs along which the disk $\Sigma_{n}$ has vertical tangent planes. These curves converge $C^{1}$ to the intersection of the $x_{3}$-axis with $\mathbb{B}(3)$. Finally there exists a disk $\mathcal{D}_{n}^{\beta}$ component of a set $\mathcal{C}_{n}^{\beta}$ (see Eq. (3)) that contains the intrinsic ball $\bar{B}_{\Sigma_{n}}(\mathbf{0}, 1 / n)$ and contains a component $\gamma(n)$ of $\Gamma_{n} \cap \mathcal{C}_{n}^{\beta} \subset \mathbb{B}(1+2 \beta), \beta \in\left(1, \frac{1}{3}\right)$. Recall that by taking $n$ sufficiently large, $\beta$ can be taken arbitrarily small.

In order for it to be easier to apply the arguments in the proof of part B of Theorem 1.1, we will soon translate the surfaces $\Sigma_{n}$ so that the translations of the curves $\gamma(n)$ contain the origin.

First recall from the proof of part B of Theorem 1.1 that we can view $\mathcal{D}_{n}^{\beta}$ to be ruled by curves $\alpha_{t}^{n}$, each of which intersects $\gamma(n)$ in a single point with $x_{3}$-coordinate equal to $t$. Let $p_{n} \in \gamma(n)$ be the point such that $\mathbf{0} \in \alpha_{x_{3}\left(p_{n}\right)}^{n}$ and consider the translated surfaces $\widetilde{\Sigma}_{n}=\Sigma_{n}-p_{n}$ so that $p_{n}$ is sent to the origin, the origin is sent to $q_{n}:=-p_{n}$ and $y_{n}$ is sent to $z_{n}:=y_{n}-p_{n}$. Abusing the notation, we use the same notation for the translation of $\gamma(n)$ and $\mathcal{D}_{n}^{\beta}$. Let $G_{n}^{\beta}$ be the map as defined in Eq. (6) of the previous section, but restricted to $\mathcal{D}_{n}^{\beta} \cap \mathbb{B}\left(\frac{1}{2}\right)$. Note that since $q_{n} \in \alpha_{0}^{n}, x_{3}\left(G_{n}^{\beta}\left(q_{n}\right)\right)=0$. Let $Q_{n}=G_{n}^{\beta}\left(q_{n}\right)$ and $Z_{n}=G_{n}^{\beta}\left(z_{n}\right)$. In this new setting, Eq. (12) becomes

$$
\operatorname{dist}_{\widetilde{\Sigma}_{n}}\left(z_{n}, q_{n}\right) \geq(1+\delta)\left(\sqrt{2}\left|z_{n}-q_{n}\right|+2 r_{n}\right) .
$$

We will obtain a contradiction by estimating $\operatorname{dist}_{\widetilde{\Sigma}_{n}}\left(z_{n}, q_{n}\right)$ from above.

Recall from the previous section the definitions of $c_{z_{n}, q_{n}}^{\beta, n}$ and $C_{z_{n}, q_{n}}^{\beta, n}$, that

$$
\operatorname{dist}_{\widetilde{\Sigma}_{n}}\left(z_{n}, q_{n}\right) \leq \operatorname{Length}\left(c_{z_{n}, q_{n}}^{\beta, n}\right) \leq(1+\beta)^{2} \operatorname{Length}\left(C_{z_{n}, q_{n}}^{\beta, n}\right),
$$


and that by taking $n$ sufficiently large, $\beta$ can be taken arbitrarily small. This, together with Eq. (13) gives that

$$
(1+\delta)\left(\sqrt{2}\left|z_{n}-q_{n}\right|+2 r_{n}\right) \leq(1+\beta)^{2} \operatorname{Length}\left(C_{z_{n}, q_{n}}^{\beta, n}\right) \leq(1+\delta)^{\frac{1}{2}} \operatorname{Length}\left(C_{z_{n}, q_{n}}^{\beta, n}\right),
$$

if $(1+\beta)^{2} \leq(1+\delta)^{\frac{1}{2}}$. In other words,

$$
(1+\delta)^{\frac{1}{2}}\left(\sqrt{2}\left|z_{n}-q_{n}\right|+2 r_{n}\right) \leq \operatorname{Length}\left(C_{z_{n}, q_{n}}^{\beta, n}\right),
$$

By working with the distance $D$ in Definition 3.6, it can be shown that if there exists $t>0$ such that Length $\left(C_{z_{n}, q_{n}}^{\beta, n}\right)=t+2\left|Q_{n}\right|$, then $\left|Q_{n}-Z_{n}\right| \geq \frac{\sqrt{2}}{2} t$, see Fig. 2. Note that since $x_{3}\left(Q_{n}\right)=0$, then $\left|Q_{n}\right|$ is equal to the distance from $Q_{n}$ to the $x_{3}$-axis. In order to prove that such a positive $t$ exists, it will suffice to show that for $n$ large, Length $\left(C_{z_{n}, q_{n}}^{\beta, n}\right)-2\left|Q_{n}\right|>0$. For the time being, let us assume that such a positive $t$ exists. Then, by the previous discussion, $t \leq \sqrt{2}\left|Q_{n}-Z_{n}\right|$ and thus

$$
\operatorname{Length}\left(C_{z_{n}, q_{n}}^{\beta, n}\right)=t+2\left|Q_{n}\right| \leq \sqrt{2}\left|Q_{n}-Z_{n}\right|+2\left|Q_{n}\right|
$$

This, together with inequality (14), implies that

$$
(1+\delta)^{\frac{1}{2}}\left(\sqrt{2}\left|z_{n}-q_{n}\right|+2 r_{n}\right) \leq \sqrt{2}\left|Q_{n}-Z_{n}\right|+2\left|Q_{n}\right|
$$

Claim 4.5 Given $\mu>0$ there exists $N(\mu)>0$ such that for any $n \geq N(\mu)$, $\left|Q_{n}\right| \leq(1+\mu) r_{n}$.

Proof Arguing by contradiction, assume that there exists $\mu>0$ such that $\left|Q_{n}\right| \geq(1+\mu) r_{n}$. By the arguments in Sect. 3,

$$
\operatorname{dist}_{\widetilde{\Sigma}_{n}}\left(\mathbf{0}, q_{n}\right) \leq(1+\beta)\left|Q_{n}\right| \leq 2\left|Q_{n}\right| \text { and } \sup _{B_{\widetilde{\Sigma}_{n}}\left(q_{n},\left(1-\frac{\sqrt{2}}{2}\right) r_{n}\right)}\left|A_{\widetilde{\Sigma}_{n}}\right| \geq r_{n}^{-1} \text {. }
$$

Consider the sequence of rescaled surfaces $\Sigma_{n}^{\prime}$ given by $\frac{1}{\left|Q_{n}\right|} \widetilde{\Sigma}_{n}$ and let $q_{n}^{\prime}=\frac{1}{\left|Q_{n}\right|} q_{n}, Q_{n}^{\prime}=\frac{1}{\left|Q_{n}\right|} Q_{n}$. Note that $\operatorname{dist}_{\Sigma_{n}^{\prime}}\left(\mathbf{0}, q_{n}^{\prime}\right) \leq 2$. Since $Q_{n}^{\prime}$ is the projection of $q_{n}^{\prime}$ to the $\left(x_{1}, x_{2}\right)$-plane, the Euclidean distance from $q_{n}^{\prime}$ to the $x_{3}$-axis is equal to 1 while the distance from $q_{n}^{\prime}$ to the $\left(x_{1}, x_{2}\right)$-plane is bounded. Note also that

$$
\sup _{B_{\Sigma_{n}^{\prime}}\left(q_{n}^{\prime},\left(1-\frac{\sqrt{2}}{2}\right) \frac{r_{n}}{\left|Q_{n}\right|}\right)}\left|A_{\Sigma_{n}^{\prime}}\right| \geq \frac{\left|Q_{n}\right|}{r_{n}} \geq 1+\mu
$$

and the tangent plane to $\Sigma_{n}^{\prime}$ at $\mathbf{0}$ is vertical. 
By Theorem 1.1, see also the arguments in Claim 3.4, after going to a subsequence, $\Sigma_{n}^{\prime}$ converges either to a vertical helicoid containing the $x_{3^{-}}$ axis or to a foliation of $\mathbb{R}^{3}$ minus the $x_{3}$-axis by horizontal planes. Since $\sup _{B_{\Sigma_{n}^{\prime}}\left(q_{n}^{\prime},\left(1-\frac{\sqrt{2}}{2}\right) \frac{r_{n}}{\left|Q_{n}\right|} \mid\right.}\left|A_{\Sigma_{n}^{\prime}}\right| \geq 1+\mu, \frac{r_{n}}{\left|Q_{n}\right|} \leq \frac{1}{1+\mu}<1$, and the Euclidean distance from $q_{n}^{\prime}$ to the $x_{3}$-axis is equal to $1, \Sigma_{n}^{\prime}$ must converge to a vertical helicoid $\mathcal{H}$ containing the $x_{3}$-axis. Let

$$
\lim _{n \rightarrow \infty} \frac{\left|Q_{n}\right|}{r_{n}}=: T \in[1+\mu, \infty) .
$$

By the previous discussion and the definitions of $q_{n}^{\prime}$ and $Q_{n}^{\prime}, \lim _{n \rightarrow \infty} q_{n}^{\prime}$ exists and it is equal to $Q:=\lim _{n \rightarrow \infty} Q_{n}^{\prime}$. Note that

$$
\sup _{B_{\mathcal{H}}\left(Q,\left(1-\frac{\sqrt{2}}{2}\right) / T\right)}\left|A_{\mathcal{H}}\right| \geq T \geq 1+\mu
$$

$|Q|=1$ and $x_{3}(Q)=0$. Thus there exists a point $P \in \bar{B}_{\mathcal{H}}\left(Q,\left(1-\frac{\sqrt{2}}{2}\right) / T\right)$ with $\left|A_{\mathcal{H}}\right|(P) \geq T>1$ with its distance from the $x_{3}$-axis at least $1-\left(1-\frac{\sqrt{2}}{2}\right) /$ $T>\frac{\sqrt{2}}{2}$. This contradicts the geometric property that on a vertical helicoid containing the $x_{3}$-axis, points of distance greater than $\frac{\sqrt{2}}{2}$ from the $x_{3}$-axis must have norm of the second fundamental form less than 1 . This finishes the proof of the claim.

By taking $\mu$ such that $1+\mu \leq(1+\delta)^{\frac{1}{2}}-\rho$, for a fixed $\rho \in\left(0,(1+\delta)^{\frac{1}{2}}-1\right)$ we have obtained that for $n$ sufficiently large

$$
\begin{aligned}
& (1+\delta)^{\frac{1}{2}}\left(\sqrt{2}\left|z_{n}-q_{n}\right|+2 r_{n}\right) \leq \sqrt{2}\left|Q_{n}-Z_{n}\right|+2\left|Q_{n}\right| \\
& \quad \leq \sqrt{2}\left|Q_{n}-Z_{n}\right|+2\left((1+\delta)^{\frac{1}{2}}-\rho\right) r_{n} .
\end{aligned}
$$

In other words,

$$
(1+\delta)^{\frac{1}{2}} \sqrt{2}\left|z_{n}-q_{n}\right| \leq \sqrt{2}\left|Q_{n}-Z_{n}\right|-2 \rho r_{n}
$$

Next we use Claim 4.5 to prove that $\operatorname{Length}\left(C_{z_{n}, q_{n}}^{\beta, n}\right)-2\left|Q_{n}\right|>0$. By Eq. (14),

$$
(1+\delta)^{\frac{1}{2}} 2 r_{n} \leq \operatorname{Length}\left(C_{z_{n}, q_{n}}^{\beta, n}\right)
$$


Therefore, using this, Claim 4.5 and our choice of $\mu$ we have that if $n$ is sufficiently large,

$$
\operatorname{Length}\left(C_{z_{n}, q_{n}}^{\beta, n}\right)-2\left|Q_{n}\right| \geq(1+\delta)^{\frac{1}{2}} 2 r_{n}-(1+\mu) 2 r_{n} \geq 2 \rho r_{n}>0 .
$$

In order to finish the proof, we argue similarly to the proof of Claim 4.5. After passing to a subsequence, we can assume that one of the following three cases holds.

1. $\lim _{n \rightarrow \infty} \frac{r_{n}}{\left|z_{n}\right|}=L \in(0, \infty)$;

2. $\lim _{n \rightarrow \infty} \frac{r_{n}}{\left|z_{n}\right|}=0$;

3. $\lim _{n \rightarrow \infty} \frac{r_{n}}{\left|z_{n}\right|}=\infty$.

First consider the case where $\lim _{n \rightarrow \infty} \frac{r_{n}}{\left|z_{n}\right|}=L \in(0, \infty)$. Consider the sequence of rescaled surfaces $\Sigma_{n}^{\prime}$ given by $\frac{1}{\left|z_{n}\right|} \widetilde{\Sigma}_{n}$ and let $q_{n}^{\prime}=\frac{1}{\left|z_{n}\right|} q_{n}, Q_{n}^{\prime}=$ $\frac{1}{\left|z_{n}\right|} Q_{n}, z_{n}^{\prime}=\frac{1}{\left|z_{n}\right|} z_{n}, Z_{n}^{\prime}=\frac{1}{\left|z_{n}\right|} Z_{n}$.

By Eqs. (15), (16) and Claim 4.5, we then have

$$
\begin{aligned}
& d_{\Sigma_{n}^{\prime}}\left(q_{n}^{\prime}, \mathbf{0}\right) \leq(1+\beta)\left|Q_{n}^{\prime}\right| \leq(1+\beta)(1+\mu) \frac{r_{n}}{\left|z_{n}\right|}, \\
& \sup _{B_{\Sigma_{n}^{\prime}}\left(q_{n}^{\prime},\left(1-\frac{\sqrt{2}}{2}\right) \frac{r_{n}}{|z n|}\right)}\left|A_{\Sigma_{n}^{\prime}}\right| \geq \frac{\left|z_{n}\right|}{r_{n}}, \\
& (1+\delta)^{\frac{1}{2}} \sqrt{2}\left|z_{n}^{\prime}-q_{n}^{\prime}\right| \leq \sqrt{2}\left|Q_{n}^{\prime}-Z_{n}^{\prime}\right|-2 \rho \frac{r_{n}}{\left|z_{n}\right|} .
\end{aligned}
$$

Notice that after replacing by a subsequence, the sequence of points $z_{n}^{\prime}$ converges to a point on the unit sphere.

By Theorem 1.1, see also the arguments in Claim 3.4, and the fact that $\lim _{n \rightarrow \infty} \frac{\left|z_{n}\right|}{r_{n}}=\frac{1}{L}>0$, a subsequence of $\Sigma_{n}^{\prime}$ converges either to a vertical helicoid containing the $x_{3}$-axis or to a foliation of $\mathbb{R}^{3}$ minus the $x_{3}$-axis by horizontal planes. (Note that when $\lim _{n \rightarrow \infty} \frac{r_{n}}{\left|z_{n}\right|}=0$, in which case $\lim _{n \rightarrow \infty} \frac{\left|z_{n}\right|}{r_{n}}=\infty$, the surfaces $\Sigma_{n}^{\prime}$ converge to a foliation of $\mathbb{R}^{3}$ minus the $x_{3}$-axis by horizontal planes.)

In either case, $\lim _{n \rightarrow \infty} z_{n}^{\prime}$ exists and it is equal to $\lim _{n \rightarrow \infty} Z_{n}^{\prime}$. Similarly, $\lim _{n \rightarrow \infty} q_{n}^{\prime}$ exists and it is equal to $\lim _{n \rightarrow \infty} Q_{n}^{\prime}$. In particular, $\lim _{n \rightarrow \infty}\left|z_{n}^{\prime}-q_{n}^{\prime}\right|$ exists and it is equal to $\lim _{n \rightarrow \infty}\left|Z_{n}^{\prime}-Q_{n}^{\prime}\right|$. Thus, since

$$
\lim _{n \rightarrow \infty} 2 \rho \frac{r_{n}}{\left|z_{n}\right|}=2 \rho L>0
$$


by choosing $n$ sufficiently large, one contradicts the inequality

$$
(1+\delta)^{\frac{1}{2}} \sqrt{2}\left|z_{n}^{\prime}-q_{n}^{\prime}\right| \leq \sqrt{2}\left|Q_{n}^{\prime}-Z_{n}^{\prime}\right|-2 \rho \frac{r_{n}}{\left|z_{n}\right|} .
$$

Suppose that $\lim _{n \rightarrow \infty} \frac{r_{n}}{\left|z_{n}\right|}=0$. This being the case, in order to obtain a contradiction, we argue exactly as in the previous case and note that $\lim _{n \rightarrow \infty}\left|z_{n}^{\prime}-q_{n}^{\prime}\right|=1 \neq 0$ since $\lim _{n \rightarrow \infty} q_{n}^{\prime}=\mathbf{0}$ and $z_{n}^{\prime}$ converges to a point on the unit sphere. Thus, by choosing $n$ sufficiently large, one again contradicts the inequality

$$
(1+\delta)^{\frac{1}{2}} \sqrt{2}\left|z_{n}^{\prime}-q_{n}^{\prime}\right| \leq \sqrt{2}\left|Q_{n}^{\prime}-Z_{n}^{\prime}\right|-2 \rho \frac{r_{n}}{\left|z_{n}\right|} .
$$

It remains to obtain a contradiction when $\lim _{n \rightarrow \infty} \frac{r_{n}}{\left|z_{n}\right|}=\infty$. In this case, consider the sequence of rescaled surfaces $\frac{1}{r_{n}} \widetilde{\Sigma}_{n}$. Abusing the notation, let $\Sigma_{n}^{\prime}$ denote this sequence and let $q_{n}^{\prime}=\frac{1}{r_{n}} q_{n}, Q_{n}^{\prime}=\frac{1}{r_{n}} Q_{n}, z_{n}^{\prime}=\frac{1}{r_{n}} z_{n}, Z_{n}^{\prime}=\frac{1}{r_{n}} Z_{n}$. By Eqs. (15), (16) and Claim 4.5, we then have

$$
\begin{aligned}
& d_{\Sigma_{n}^{\prime}}\left(q_{n}^{\prime}, \mathbf{0}\right) \leq(1+\beta)\left|Q_{n}^{\prime}\right| \leq(1+\beta)(1+\mu), \\
& \quad \sup \left|A_{\Sigma_{n}^{\prime}}\right| \geq 1, \\
& B_{\Sigma_{n}^{\prime}}\left(q_{n}^{\prime}, 1-\frac{\sqrt{2}}{2}\right) \\
& (1+\delta)^{\frac{1}{2}} \sqrt{2}\left|z_{n}^{\prime}-q_{n}^{\prime}\right| \leq \sqrt{2}\left|Q_{n}^{\prime}-Z_{n}^{\prime}\right|-2 \rho .
\end{aligned}
$$

Arguing like in the previous case, by Theorem 1.1 a subsequence of the surfaces $\Sigma_{n}^{\prime}$ converges either to a vertical helicoid containing the $x_{3}$-axis or to a foliation of $\mathbb{R}^{3}$ minus the $x_{3}$-axis by horizontal planes. In either case, since $\lim _{n \rightarrow \infty} \frac{r_{n}}{\left|z_{n}\right|}=\infty$, then $\left|z_{n}^{\prime}\right|=\frac{\left|z_{n}\right|}{r_{n}}$ is bounded from above, and it follows that $\lim _{n \rightarrow \infty} z_{n}^{\prime}$ exists and it is equal to $\lim _{n \rightarrow \infty} Z_{n}^{\prime}$. Similarly, $\lim _{n \rightarrow \infty} q_{n}^{\prime}$ exists and it is equal to $\lim _{n \rightarrow \infty} Q_{n}^{\prime}$. In particular $\lim _{n \rightarrow \infty}\left|z_{n}^{\prime}-q_{n}^{\prime}\right|$ exists and it is equal to $\lim _{n \rightarrow \infty}\left|Z_{n}^{\prime}-Q_{n}^{\prime}\right|$. Therefore, similarly to the previous two cases, by choosing $n$ sufficiently large, one contradicts the inequality

$$
(1+\delta)^{\frac{1}{2}} \sqrt{2}\left|z_{n}^{\prime}-q_{n}^{\prime}\right| \leq \sqrt{2}\left|Q_{n}^{\prime}-Z_{n}^{\prime}\right|-2 \rho .
$$

This finishes the proof of the theorem.

\subsection{Curvature estimates}

In this section we prove three other useful corollaries of Theorem 2.5 and Corollary 2.6. The next corollary essentially states that if three $H$-disks are 
sufficiently close to a point that is away from their boundaries, then nearby that point, the surfaces satisfy a curvature estimate.

Corollary 4.6 There exist constants $\varepsilon \in(0,1), C>1$ such that the following holds. Let $\Sigma_{1}, \Sigma_{2}, \Sigma_{3}$ be three pairwise disjoint $H_{i}$-disks with $\partial \Sigma_{i} \subset\left[\mathbb{R}^{3} \backslash\right.$ $\mathbb{B}(1)]$ for $i=1,2$, 3. If $\mathbb{B}(\varepsilon) \cap \Sigma_{i} \neq \varnothing$ for $i=1,2,3$, then

$$
\sup _{\mathbb{B}(\varepsilon) \cap \Sigma_{i}, i=1,2,3}\left|A_{\Sigma_{i}}\right| \leq C .
$$

Proof Arguing by contradiction, suppose that the corollary fails. In this case, for $i=1,2,3$, there exist sequences of $H_{i}(n)$-disks $\Sigma_{i}(n)$ with $\partial \Sigma_{i}(n) \subset$ $\partial \mathbb{B}(1)$ such that $\mathbb{B}\left(\frac{1}{n^{2}}\right) \cap \Sigma_{i} \neq \varnothing$ but

$$
\sup _{\mathbb{B}\left(\frac{1}{n^{2}}\right) \cap\left[\cup_{i=1}^{3} \Sigma_{i}(n)\right]}\left|A_{\cup_{i=1}^{3} \Sigma_{i}(n)}\right|>n^{2} .
$$

Consider the sequence of rescaled surfaces $M_{i}(n)=n \Sigma_{i}(n), i=1,2,3$, and note that $M_{i}(n) \cap \mathbb{B}(1 / n) \neq \varnothing$ for each $i$. Without loss of generality, we can assume that

$$
\sup _{\mathbb{B}(1 / n) \cap M_{1}(n)}\left|A_{M_{1}(n)}\right|>n .
$$

After replacing by a subsequence, Theorem 1.1 implies that the sequence $M_{1}(n)$ converges $C^{\alpha}, \alpha \in(0,1)$, to a foliation $\mathcal{F}$ of $\mathbb{R}^{3} \backslash \mathcal{S}_{1}$ by parallel planes, where $\mathcal{S}_{1}$ is a line orthogonal to the planes; without loss of generality we will henceforth assume that the parallel planes are horizontal.

Since for $i=2,3, M_{i}(n) \cap \mathbb{B}(1 / n) \neq \varnothing$, Theorem 1.1, Corollary 2.6 and the embeddedness of the disconnected surface $\cup_{i=1}^{3} M_{i}(n)$ imply that, after replacing by new subsequences, each of the sequences $M_{i}(n)$ converges $C^{\alpha}$, for $\alpha \in(0,1)$, to the foliation $\mathcal{F}$ of $\mathbb{R}^{3} \backslash \mathcal{S}_{1}$ by horizontal planes. It follows that for $n$ large, each of the surfaces $M_{i}, i=1,2,3$, yields at least one related analytic arc component in the set $\Gamma_{n}$ where $\cup_{i=1}^{3} M_{i}(n)$ has vertical tangent planes in $\mathbb{B}(3)$. But the total number of such components in $\Gamma_{n}$ must be at most two by adapting the arguments in the proof of Claim 3.4. This gives a contradiction which completes the proof of the claim.

From Corollary 4.6 one easily obtains the result below.

Corollary 4.7 There exists $\varepsilon \in(0,1)$ such that the following holds. Let $\Sigma_{1}$, $\Sigma_{2}, \Sigma_{3}$ be $H_{i}$-disks with $\partial \Sigma_{i} \subset \partial \mathbb{B}(1)$ for $i=1,2$, 3. If $\mathbb{B}(\varepsilon) \cap \Sigma_{i} \neq \varnothing$ for $i=1,2,3$, then, after a rotation, each component of $\Sigma_{i} \cap \mathbb{B}(2 \varepsilon)$ intersecting 
$\mathbb{B}(\varepsilon)$ is a graph with norm of its gradient bounded by 1 over its projection into the plane $\left\{x_{3}=0\right\}$.

Remark 4.8 In Corollary 0.4 of [8], Colding and Minicozzi gave an analogous result to the statement of Corollary 4.6. In their case, it suffices to have two embedded minimal disks to obtain a curvature estimate. This is because one can insert a stable minimal disk, satisfying curvature estimates, in between the two minimal disks and apply a result analogous to Corollary 2.6 for minimal disks. In our case this approach does not work and indeed the curvature estimate is not true for just two $H$-disks.

In the next proposition, we use the approach described in the previous remark to obtain a curvature estimate when certain topological conditions are satisfied.

Corollary 4.9 There exist constants $\varepsilon \in(0,1 / 2), C>1$ such that the following holds. Let $\Sigma_{1}, \Sigma_{2}$ be two pairwise disjoint $H_{i}$-disks with $\partial \Sigma_{i} \subset\left[\mathbb{R}^{3} \backslash \mathbb{B}(1)\right]$ for $i=1,2$, and $0 \leq H_{1} \leq H_{2}$. Let $W$ denote a component of $\mathbb{B}(1) \backslash\left[\Sigma_{1} \cup \Sigma_{2}\right]$ such that the mean curvature vector of $\Sigma_{2}$ points into $W$. If $W \cap \mathbb{B}(\varepsilon)$ contains a component $W^{\prime}$ such that $\Sigma_{i} \cap \partial W^{\prime} \neq \varnothing$ for $i=1,2$, then

$$
\sup _{\mathbb{B}(\varepsilon) \cap \Sigma_{i}, i=1,2}\left|A_{\Sigma_{i}}\right| \leq C .
$$

Proof By Corollary 2.6, there exists $\varepsilon>0$ such that the following holds: if $M \subset W$ is a surface such that $M \cap \mathbb{B}(\varepsilon) \neq \varnothing, \partial M \subset \partial \mathbb{B}(1)$ and $M$ satisfies a uniform estimate on the norm of its second fundamental form in $\mathbb{B}(1 / 2)$ that does not depend on $\Sigma_{1}$ or $\Sigma_{2}$, then the corollary holds. Therefore, it suffice to construct such an $M$. If $H_{2} \geq 1$, then by the extrinsic curvature estimates in Theorem 2.4, $\Sigma_{2}$ can be chosen to be such $M$. Therefore, we can assume that $\mathrm{H}_{2}<1$.

Under our hypothesis, there exists a compact, oriented, weakly stable $H_{1}$ surface $M_{1} \subset W$ such that $\partial M_{1}=\partial \Sigma_{1} \cap \partial W$ and $M_{1}$ is homologous to $\Sigma_{1} \cap \partial W$ in $W$, see for instance [1] for this construction. By the results in [13] and standard rescaling arguments, $M_{1}$ satisfies a uniform estimate on the norm of the second fundamental form in $\mathbb{B}(1 / 2)$. Since $M_{1}$ is homologous to $\Sigma_{1} \cap \partial W$ in $W$ and $W \cap \mathbb{B}(\varepsilon)$ contains a component $W^{\prime}$ such that $\Sigma_{i} \cap \partial W^{\prime} \neq \varnothing$ for $i=1,2$, then $M_{1} \cap \mathbb{B}(\varepsilon) \neq \varnothing$. This finishes the proof of the corollary.

Open Access This article is licensed under a Creative Commons Attribution 4.0 International License, which permits use, sharing, adaptation, distribution and reproduction in any medium or format, as long as you give appropriate credit to the original author(s) and the source, provide a link to the Creative Commons licence, and indicate if changes were made. The images or other third party material in this article are included in the article's Creative Commons licence, unless 
indicated otherwise in a credit line to the material. If material is not included in the article's Creative Commons licence and your intended use is not permitted by statutory regulation or exceeds the permitted use, you will need to obtain permission directly from the copyright holder. To view a copy of this licence, visit http://creativecommons.org/licenses/by/4.0/.

\section{References}

1. Alencar, H., Rosenberg, H.: Some remarks on the existence of hypersurfaces of constant mean curvature with a given boundary, or asymptotic boundary in hyperbolic space. Bull. Sci. Math. 121(1), 61-69 (1997)

2. Bernstein, J., Breiner, C.: Helicoid-like minimal disks and uniqueness. J. Reine Angew. Math. 655, 129-146 (2011)

3. Bernstein, J., Tinaglia, G.: Topological type of limit laminations of embedded minimal disks. J. Differ. Geom. 102(1), 1-23 (2016). arXiv:1309.6260

4. Choi, T., Meeks, W.H., III., White, B.: A rigidity theorem for properly embedded minimal surfaces in $\mathbb{R}^{3}$. J. Differ. Geom. 32, 65-76 (1990)

5. Colding, T.H., Minicozzi, W.P., II.: The space of embedded minimal surfaces of fixed genus in a 3-manifold I; estimates off the axis for disks. Ann. Math. 160, 27-68 (2004)

6. Colding, T.H., Minicozzi, W.P., II.: The space of embedded minimal surfaces of fixed genus in a 3-manifold II; multi-valued graphs in disks. Ann. Math. 160, 69-92 (2004)

7. Colding, T.H., Minicozzi, W.P., II.: The space of embedded minimal surfaces of fixed genus in a 3-manifold III; planar domains. Ann. Math. 160, 523-572 (2004)

8. Colding, T.H., Minicozzi, W.P., II.: The space of embedded minimal surfaces of fixed genus in a 3-manifold IV; locally simply-connected. Ann. Math. 160, 573-615 (2004)

9. Colding, T.H., Minicozzi, W.P., II.: The Calabi-Yau conjectures for embedded surfaces. Ann. Math. 167, 211-243 (2008)

10. Hoffman, D., Meeks, W.H., III.: The strong halfspace theorem for minimal surfaces. Invent. Math. 101, 373-377 (1990)

11. Korevaar, N., Kusner, R., Solomon, B.: The structure of complete embedded surfaces with constant mean curvature. J. Differ. Geom. 30, 465-503 (1989)

12. Kusner, R.: Global geometry of extremal surfaces in three-space. Ph.D. thesis, University of California, Berkeley (1988)

13. López, F.J., Ros, A.: Complete minimal surfaces of index one and stable constant mean curvature surfaces. Comment. Math. Helv. 64, 34-53 (1989)

14. Meeks, W.H., III.: The regularity of the singular set in the Colding and Minicozzi lamination theorem. Duke Math. J. 123(2), 329-334 (2004)

15. Meeks, W.H., III.: The limit lamination metric for the Colding-Minicozzi minimal lamination. Ill. J. Math. 49(2), 645-658 (2005)

16. Meeks, W.H., III., Pérez, J., Ros, A.: The geometry of minimal surfaces of finite genus I; curvature estimates and quasiperiodicity. J. Differ. Geom. 66, 1-45 (2004)

17. Meeks, W.H., III., Pérez, J., Ros, A.: Stable constant mean curvature surfaces. In: Ji, Lizhen, Li, Peter, Schoen, Richard, Simon, Leon (eds.) Handbook of Geometrical Analysis, vol. 1, pp. 301-380. International Press, Vienna (2008)

18. Meeks, W.H., III., Pérez, J., Ros, A.: Limit leaves of an H lamination are stable. J. Differ. Geom. 84(1), 179-189 (2010)

19. Meeks, W.H., III., Pérez, J., Ros, A.: Structure theorems for singular minimal laminations. J. Reine Angew. Math. 763, 271-312 (2020)

20. Meeks, W.H., III., Rosenberg, H.: The uniqueness of the helicoid. Ann. Math. 161(2), 727-758 (2005)

21. Meeks, W.H., III., Rosenberg, H.: The minimal lamination closure theorem. Duke Math. J. 133(3), 467-497 (2006) 
22. Meeks, W.H., III., Tinaglia, G.: Chord arc properties for constant mean curvature disks. J. Geom. Topol. 22(1), 305-322 (2018). arXiv:1408.5578.pdf

23. Meeks, W.H., III., Tinaglia, G.: Curvature estimates for constant mean curvature surfaces. Duke Math. J. 168, 3057-3102 (2019). arXiv:1502.06110

24. Meeks, W.H., III., Tinaglia, G.: One-sided curvature estimates for $H$-disks. Camb. J. Math. 8(3), 479-503 (2020). arXiv:1408.5233

25. Smyth, B., Tinaglia, G.: The number of constant mean curvature isometric immersions of a surface. Comment. Math. Helv. 88(1), 163-183 (2013)

26. White, B.: Curvatures of embedded minimal disks blow up on subsets of $C^{1}$ curves. J. Differ. Geom. 100(2), 389-394 (2015)

Publisher's Note Springer Nature remains neutral with regard to jurisdictional claims in published maps and institutional affiliations. 